\title{
Do Reaction Conditions Affect the Stereoselectivity in the Staudinger Reaction?
}

\author{
Yikai Wang, Yong Liang, Lei Jiao, Da-Ming Du, and Jiaxi Xu* \\ Beijing National Laboratory for Molecular Sciences (BNLMS), Key Laboratory of Bioorganic Chemistry \\ and Molecular Engineering of Ministry of Education, College of Chemistry and Molecular Engineering, \\ Peking University, Beijing 100871, People's Republic of China \\ jxxu@pku.edu.cn
}

\section{Supplemental Materials}

\section{Content} S1

Experimental Details . S2

Analytic Data of the Unknown Products. S4

Copies of ${ }^{1} \mathrm{H}$ and ${ }^{13} \mathrm{C}$ NMR Spectra of the Unknown Products S10 


\section{Experimental Details}

General. $\alpha$-Diazocarbonyl compounds $\mathbf{1}^{1}$ and $\mathbf{5} ;^{2}$ and imines $\mathbf{2 a - \mathbf { f } ^ { 3 , 4 }}$ were prepared according to published procedures. Toluene, cyclohexane, octane, and 1,2-dimethoxyethane were refluxed with sodium and freshly distilled prior to use. Acetonitrile, $o$-dichlorobenzene, and 1,2-dichloroethane were refluxed with calcium hydride and freshly distilled prior to use. All reactions were performed under a nitrogen atmosphere. ${ }^{1} \mathrm{H}$ and ${ }^{13} \mathrm{C}$ NMR spectra were recorded in $\mathrm{CDCl}_{3}$ with $\mathrm{TMS}$ as an internal standard. All known products show the identical analytic data to reported data in the literatures. ${ }^{4,5,6,7}$

\section{Determination of the cis/trans Ratios of the Crude $\beta$-Lactam Products.}

1. For the reactions of $S$-phenyl 2-diazoethanethioate (1) and phenylthioacetyl chloride (6a) with imines $\mathbf{2}$, the cis/trans ratios were obtained by the integral of the corresponding $C(3)$ and $C(4)$ protons of $\beta$-lactams 3 and 4 in ${ }^{1} \mathrm{H}$ NMR spectra (protons of $c i s$-isomers appear at about 4.7 and 4.9 ppm, while protons of trans-isomers appear at about 4.0 and $4.3 \mathrm{ppm}$ ) of the crude reaction mixture.

2. For the reactions of 1-diazoacetone (5) and propionyl chloride (6b) with imines $\mathbf{2}$, the cis/trans ratios were obtained by the integral of the corresponding protons $\mathrm{C}(3)$ and $\mathrm{C}(4)$ protons of $\beta$-lactams 7 and 8 in ${ }^{1} \mathrm{H}$ NMR spectra (protons of $c i s$-isomers appear at about 3.5 and $4.8 \mathrm{ppm}$, while protons of trans-isomers appear at about 2.9 and $4.1 \mathrm{ppm}$ ) of the crude reaction mixture.

3. For the reactions of phenylacetyl chloride (6c) and imines $\mathbf{2}$, the cis/trans ratios were obtained by the integral of the corresponding $\mathrm{C}(3)$ and $\mathrm{C}(4)$ protons of $\beta$-lactams 9 and $\mathbf{1 0}$ in ${ }^{1} \mathrm{H}$ NMR spectra (protons of cis-isomers appear at about 4.7 and $5.0 \mathrm{ppm}$, while protons of trans-isomers appear at about 4.1 and $4.4 \mathrm{ppm})$ of the crude reaction mixture.

4. For the reactions of phenoxyacetyl chloride (6d) and imines $\mathbf{2}$, the cis/trans ratios were obtained by the integral of the corresponding $\mathrm{C}(3)$ and $\mathrm{C}(4)$ protons of $\beta$-lactams 11 and $\mathbf{1 2}$ in ${ }^{1} \mathrm{H}$ NMR spectra (protons of cis-isomers appear at about 5.1 and 5.4 ppm, while protons of trans-isomers appear at about 4.5 and $4.9 \mathrm{ppm}$ ) of the crude reaction mixture.

5. For the reactions of phthimidoacetyl chloride (6e) and imines $\mathbf{2}$, the cis/trans ratios were obtained by the integral of the corresponding $\mathrm{C}(3)$ and $\mathrm{C}(4)$ protons of $\beta$-lactams 13 and $\mathbf{1 4}$ in ${ }^{1} \mathrm{H}$ NMR spectra (protons of $c i s$-isomers appear at about 5.1 and $5.5 \mathrm{ppm}$, while protons of trans-isomers appear at about 5.0 and $5.1 \mathrm{ppm})$ of the crude reaction mixture.

\footnotetext{
${ }^{1}$ Danheiser, R. L.; Okamoto, I.; Lawlor, M. D.; Lee, T. W. Org. Synth. Vol. 80, 160-171.

2 Bridson, J. N.; Hooz, J. Org. Synth. Coll. Vol. 6, 386.

${ }^{3}$ Linder, M. R.; Frey, W. U.; Podlech, J. J. Chem. Soc., Perkin Trans. 1 2001, 2566-2577.

${ }^{4}$ Jiao, L.; Liang, Y.; Xu, J. X. J. Am. Chem. Soc. 2006, 128, 6060-6069.

5 Ohmori, H.; Maeda, H.; Kikuoka, M.; Maki, T.; Masui, M. Tetrahedron 1991, 47, 767-776.

${ }^{6}$ Ejaegher, Y.; Denolf, B.; Stevens, C. V.; De Kimpe, N. Synthesis 2005, 193-198.

7 Abd-Elzaher, M. M.; Fischer, H. J. Organomet. Chem. 1999, 588, 235-241.
} 
6. For the reactions of chloroacetyl chloride (6f) and imines 2, the cis/trans ratios were obtained by the integral of the corresponding $\mathrm{C}(3)$ and $\mathrm{C}(4)$ protons of $\beta$-lactams 15 and 16 in ${ }^{1} \mathrm{H}$ NMR spectra (protons of cis-isomers appear at about 5.0 and $5.1 \mathrm{ppm}$, while protons of trans-isomers appear at about 4.4 and $4.5 \mathrm{ppm})$ of the crude reaction mixture. 


\section{Analytic Data for the Unknown Products}

( \pm )-cis-1-Isopropyl-3-methyl-4-(4-trifluoromethylphenyl)azetidin-2-one (7e)<smiles>CCCN1C(=O)[C@H](C)[C@H]1c1ccc(C(F)(F)F)cc1</smiles>

Yellowish oil; IR $(\mathrm{KBr}) v\left(\mathrm{~cm}^{-1}\right): 1746(\mathrm{C}=\mathrm{O}) ;{ }^{1} \mathrm{H} \mathrm{NMR}\left(300 \mathrm{MHz}, \mathrm{CDCl}_{3}\right): \delta 0.80(\mathrm{~d}, J=7.8 \mathrm{~Hz}$, $3 \mathrm{H}), 1.14(\mathrm{~d}, J=6.9 \mathrm{~Hz}, 3 \mathrm{H}), 1.33(\mathrm{~d}, J=6.6 \mathrm{~Hz}, 3 \mathrm{H}), 3.47$ (dq, $J=5.7,7.8 \mathrm{~Hz}, 1 \mathrm{H}), 3.78$ (qq, $J$ $=6.6,6.9 \mathrm{~Hz}, 1 \mathrm{H}), 4.82(\mathrm{~d}, J=5.7 \mathrm{~Hz}, 1 \mathrm{H}), 7.41(\mathrm{~d}, J=8.0 \mathrm{~Hz}, 2 \mathrm{H}), 7.65(\mathrm{~d}, J=8.0 \mathrm{~Hz}, 2 \mathrm{H}) . \mathrm{MS}$ (ESI) $m / z$ : $272\left(\mathrm{MH}^{+}\right)$; HRMS Cacld. for $\mathrm{C}_{14} \mathrm{H}_{17} \mathrm{~F}_{3} \mathrm{NO}\left(\mathrm{MH}^{+}\right)$272.1262; Found 272.1262.

( \pm )-trans-1-Isopropyl-4-(4-methoxylphenyl)-3-methylazetidin-2-one (8a)<smiles>CCCN1C(=O)[C@H](C)[C@H]1c1ccc(OC)cc1</smiles>

Yellowish oil; IR $(\mathrm{KBr}) v\left(\mathrm{~cm}^{-1}\right): 1744(\mathrm{C}=\mathrm{O}) ;{ }^{1} \mathrm{H}$ NMR (300 MHz, $\left.\mathrm{CDCl}_{3}\right): \delta 1.00(\mathrm{~d}, J=6.6$ $\mathrm{Hz}, 3 \mathrm{H}), 1.24(\mathrm{~d}, J=6.6 \mathrm{~Hz}, 3 \mathrm{H}), 1.33(\mathrm{~d}, J=7.5 \mathrm{~Hz}, 3 \mathrm{H}), 2.92(\mathrm{dq}, J=1.5,7.5 \mathrm{~Hz}, 1 \mathrm{H}), 3.77$ (heptet, $J=6.6 \mathrm{~Hz}, 1 \mathrm{H}), 3.82(\mathrm{~s}, 3 \mathrm{H}), 4.06(\mathrm{~d}, J=1.5 \mathrm{~Hz}, 1 \mathrm{H}), 6.90(\mathrm{~d}, J=8.6 \mathrm{~Hz}, 2 \mathrm{H}), 7.27$ (d, $J$ $=8.6 \mathrm{~Hz}, 2 \mathrm{H}) ;{ }^{13} \mathrm{C} \mathrm{NMR}\left(75.5 \mathrm{MHz}, \mathrm{CDCl}_{3}\right): \delta 12.8,20.3,21.2,44.5,54.1,55.3,61.3,114.1$, 127.7, 131.4, 159.6, 171.0. MS (ESI) $m / z: 234\left(\mathrm{MH}^{+}\right)$; Anal. Cacld. for $\mathrm{C}_{14} \mathrm{H}_{19} \mathrm{NO}_{2}$ (233.14): $\mathrm{C}$, 72.07; H, 8.21; N, 6.00. Found: C, 71.78; H, 8.37; N, 5.84\%.

( \pm )-trans -1 -Isopropyl-3-methyl-4-(4-methylphenyl)azetidin-2-one (8b)<smiles>CCCN1C(=O)[C@H](C)[C@H]1c1ccc(C)cc1</smiles>

Yellowish oil; IR $(\mathrm{KBr}) v\left(\mathrm{~cm}^{-1}\right): 1747(\mathrm{C}=\mathrm{O}) ;{ }^{1} \mathrm{H}$ NMR $\left(300 \mathrm{MHz}, \mathrm{CDCl}_{3}\right): \delta 1.01(\mathrm{~d}, J=6.6 \mathrm{~Hz}$, $3 \mathrm{H}), 1.25$ (d, $J=6.6 \mathrm{~Hz}, 3 \mathrm{H}), 1.33$ (d, $J=7.5 \mathrm{~Hz}, 3 \mathrm{H}), 2.36$ (s, 3H), 2.92 (dq, $J=1.5,7.5 \mathrm{~Hz}, 1 \mathrm{H}$ ), 3.76 (heptet, $J=6.6 \mathrm{~Hz}, 1 \mathrm{H}), 4.07(\mathrm{~d}, J=1.5 \mathrm{~Hz}, 1 \mathrm{H}), 7.16-7.26(\mathrm{~m}, 4 \mathrm{H}) ;{ }^{13} \mathrm{C}$ NMR $(75.5 \mathrm{MHz}$, $\left.\mathrm{CDCl}_{3}\right): \delta 12.8,20.3,21.1,21.2,44.6,54.2,61.6,126.4,129.4,136.5,138.1,171.0$. MS (ESI) $\mathrm{m} / z$ : $218\left(\mathrm{MH}^{+}\right)$; HRMS Cacld. for $\mathrm{C}_{14} \mathrm{H}_{20} \mathrm{NO}\left(\mathrm{MH}^{+}\right)$218.1545; Found 218.1543.

( \pm )-trans-1-Isopropyl-3-methyl-4-phenylazetidin-2-one (8c)<smiles>CCCN1C(=O)C(C)C1c1ccccc1</smiles>

Yellowish oil; IR $(\mathrm{KBr}) v\left(\mathrm{~cm}^{-1}\right): 1745(\mathrm{C}=\mathrm{O}) ;{ }^{1} \mathrm{H}$ NMR (300 MHz, $\left.\mathrm{CDCl}_{3}\right): \delta 1.01(\mathrm{~d}, J=6.9$ $\mathrm{Hz}, 3 \mathrm{H}), 1.26$ (d, $J=6.9 \mathrm{~Hz}, 3 \mathrm{H}), 1.34$ (d, $J=7.2 \mathrm{~Hz}, 3 \mathrm{H}), 2.95$ (dq, $J=1.5,7.2 \mathrm{~Hz}, 1 \mathrm{H}), 3.77$ 
(heptet, $J=6.9 \mathrm{~Hz}, 1 \mathrm{H}), 4.11(\mathrm{~d}, J=1.5 \mathrm{~Hz}, 1 \mathrm{H}), 7.28-7.36(\mathrm{~m}, 5 \mathrm{H}) ;{ }^{13} \mathrm{C}$ NMR $(75.5 \mathrm{MHz}$, $\mathrm{CDCl}_{3}$ ): $\delta 12.8,20.3,21.2,44.6,54.2,61.7,126.4,128.2,128.7,139.5,170.9$. MS (ESI) $\mathrm{m} / z: 204$ $\left(\mathrm{MH}^{+}\right)$; Anal. Cacld. for $\mathrm{C}_{13} \mathrm{H}_{17} \mathrm{NO}$ (326.13): C, 76.81; H, 8.43; N, 6.89. Found: C, 77.14; H, 8.62; $\mathrm{N}, 6.88 \%$.

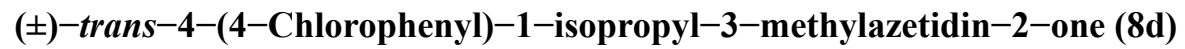<smiles>CCCN1C(=O)[C@H](C)[C@H]1c1ccc(Cl)cc1</smiles>

Yellowish oil; IR $(\mathrm{KBr}) v\left(\mathrm{~cm}^{-1}\right): 1749(\mathrm{C}=\mathrm{O}) ;{ }^{1} \mathrm{H}$ NMR $\left(300 \mathrm{MHz}, \mathrm{CDCl}_{3}\right): \delta 1.01(\mathrm{~d}, J=6.6 \mathrm{~Hz}$, $3 \mathrm{H}), 1.24(\mathrm{~d}, J=6.9 \mathrm{~Hz}, 3 \mathrm{H}), 1.34(\mathrm{~d}, J=7.5 \mathrm{~Hz}, 3 \mathrm{H}), 2.91(\mathrm{dq}, J=1.8,7.5 \mathrm{~Hz}, 1 \mathrm{H}), 3.77$ (qq, $J$ $=6.6,6.9 \mathrm{~Hz}, 1 \mathrm{H}), 4.09(\mathrm{~d}, J=1.8 \mathrm{~Hz}, 1 \mathrm{H}), 7.30(\mathrm{~d}, J=8.6 \mathrm{~Hz}, 2 \mathrm{H}), 7.35(\mathrm{~d}, J=8.6 \mathrm{~Hz}, 2 \mathrm{H}) ;{ }^{13} \mathrm{C}$ NMR (75.5 MHz, $\left.\mathrm{CDCl}_{3}\right): \delta 12.8,20.3,21.2,44.7,54.4,61.0,127.8,129.0,134.0,138.2,170.7$. MS (ESI) $m / z: 238\left(\mathrm{MH}^{+}\right)$; HRMS Cacld. for $\mathrm{C}_{13} \mathrm{H}_{17} \mathrm{ClNO}\left(\mathrm{MH}^{+}\right)$238.0999; Found 238.0996.

( \pm )-trans-1-Isopropyl-3-methyl-4-(4-trifluoromethylphenyl)azetidin-2-one (8e)<smiles>CCCN1C(=O)[C@H](C)[C@H]1c1ccc(C(F)(F)F)cc1</smiles>

Yellowish oil; IR $(\mathrm{KBr}) v\left(\mathrm{~cm}^{-1}\right): 1750(\mathrm{C}=\mathrm{O}) ;{ }^{1} \mathrm{H}$ NMR $\left(300 \mathrm{MHz}, \mathrm{CDCl}_{3}\right): \delta 1.03(\mathrm{~d}, J=6.6 \mathrm{~Hz}$, $3 \mathrm{H}), 1.26(\mathrm{~d}, J=6.6 \mathrm{~Hz}, 3 \mathrm{H}), 1.37(\mathrm{~d}, J=7.5 \mathrm{~Hz}, 3 \mathrm{H}), 2.93(\mathrm{dq}, J=1.8,7.5 \mathrm{~Hz}, 1 \mathrm{H}), 3.80$ (heptet, $J=6.6 \mathrm{~Hz}, 1 \mathrm{H}), 4.17(\mathrm{~d}, J=1.8 \mathrm{~Hz}, 1 \mathrm{H}), 7.49(\mathrm{~d}, J=8.1 \mathrm{~Hz}, 2 \mathrm{H}), 7.64(\mathrm{~d}, J=8.1 \mathrm{~Hz}, 2 \mathrm{H}) .{ }^{13} \mathrm{C}$ $\operatorname{NMR}\left(75.5 \mathrm{MHz}, \mathrm{CDCl}_{3}\right): \delta 12.8,20.4,21.2,44.9,54.6,61.1,122.1,125.81(\mathrm{~d}, J=3.5 \mathrm{~Hz}), 126.7$, 130.5 (d, $J=32.9 \mathrm{~Hz}), 143.9$, 170.7. MS (ESI) $\mathrm{m} / z$ : $272\left(\mathrm{MH}^{+}\right)$; HRMS Cacld. for $\mathrm{C}_{14} \mathrm{H}_{17} \mathrm{~F}_{3} \mathrm{NO}$ $\left(\mathrm{MH}^{+}\right)$272.1262; Found 272.1265.

( \pm )-cis-1-Isopropyl-4-(4-methoxylphenyl)-3-phenylazetidin-2-one (9a)<smiles>CCCN1C(=O)[C@H](c2ccccc2)[C@H]1c1ccc(OC)cc1</smiles>

Yellowish oil; IR $(\mathrm{KBr}) v\left(\mathrm{~cm}^{-1}\right): 1743(\mathrm{C}=\mathrm{O}) ;{ }^{1} \mathrm{H}$ NMR $\left(300 \mathrm{MHz}, \mathrm{CDCl}_{3}\right): \delta 1.12(\mathrm{~d}, J=6.6 \mathrm{~Hz}$, $3 \mathrm{H}), 1.34$ (d, $J=6.9 \mathrm{~Hz}, 3 \mathrm{H}), 3.69$ (s, 3H), 3.86 (qq, $J=6.6,6.9 \mathrm{~Hz}, 1 \mathrm{H}), 4.73$ (d, $J=5.6 \mathrm{~Hz}, 1 \mathrm{H}$ ), $4.98(\mathrm{~d}, J=5.6 \mathrm{~Hz}, 1 \mathrm{H}), 6.64(\mathrm{~d}, J=8.2 \mathrm{~Hz}, 2 \mathrm{H}), 6.96(\mathrm{~d}, J=8.2 \mathrm{~Hz}, 2 \mathrm{H}), 7.01-7.14(\mathrm{~m}, 5 \mathrm{H}) . \mathrm{MS}$ (ESI) $m / z: 296\left(\mathrm{MH}^{+}\right)$; HRMS Cacld. for $\mathrm{C}_{19} \mathrm{H}_{22} \mathrm{NO}_{2}\left(\mathrm{MH}^{+}\right)$296.1651; Found 296.1653. 
( \pm --trans-1-Isopropyl-4-(4-methoxylphenyl)-3-phenylazetidin-2-one (10a)<smiles>CCCN1C(=O)[C@@H](c2ccccc2)[C@H]1c1ccc(OC)cc1</smiles>

Colorless oil; IR (KBr) $v\left(\mathrm{~cm}^{-1}\right): 1747(\mathrm{C}=\mathrm{O}) ;{ }^{1} \mathrm{H}$ NMR $\left(300 \mathrm{MHz}, \mathrm{CDCl}_{3}\right): \delta 1.07(\mathrm{~d}, J=6.6 \mathrm{~Hz}$, $3 \mathrm{H}), 1.34(\mathrm{~d}, J=6.6 \mathrm{~Hz}, 3 \mathrm{H}), 3.83$ (s, 3H), 3.87 (heptet, $J=6.6 \mathrm{~Hz}, 1 \mathrm{H}), 4.08(\mathrm{~d}, J=2.1 \mathrm{~Hz}, 1 \mathrm{H})$, $4.44(\mathrm{~d}, J=2.1 \mathrm{~Hz}, 1 \mathrm{H}), 6.92-7.34(\mathrm{~m}, 9 \mathrm{H}) ;{ }^{13} \mathrm{C} \mathrm{NMR}\left(75.5 \mathrm{MHz}, \mathrm{CDCl}_{3}\right): \delta 20.6,21.3,45.0$, 55.3, 62.4, 64.1, 114.3, 127.3, 127.5, 127.8, 128.8, 130.8, 135.4, 159.8, 168.2. MS (ESI) $m / z: 296$ $\left(\mathrm{MH}^{+}\right)$; HRMS Cacld. for $\mathrm{C}_{19} \mathrm{H}_{22} \mathrm{NO}_{2}\left(\mathrm{MH}^{+}\right)$296.1651; Found 296.1648.

( \pm -) trans$^{-1}$-Isopropyl-3,4-diphenylazetidin-2-one (10c)<smiles>CCCN1C(=O)[C@@H](c2ccccc2)[C@H]1c1ccccc1</smiles>

Colorless oil; IR $(\mathrm{KBr}) v\left(\mathrm{~cm}^{-1}\right): 1749(\mathrm{C}=\mathrm{O}) ;{ }^{1} \mathrm{H}$ NMR $\left(300 \mathrm{MHz}, \mathrm{CDCl}_{3}\right): \delta 1.09(\mathrm{~d}, J=6.9 \mathrm{~Hz}$, $3 \mathrm{H}), 1.35$ (d, $J=6.9 \mathrm{~Hz}, 3 \mathrm{H}), 3.87$ (heptet, $J=6.9 \mathrm{~Hz}, 1 \mathrm{H}), 4.10(\mathrm{~d}, J=2.1 \mathrm{~Hz}, 1 \mathrm{H}), 4.48$ (d, $J=$ $2.1 \mathrm{~Hz}, 1 \mathrm{H}), 7.27-7.42(\mathrm{~m}, 9 \mathrm{H}) ;{ }^{13} \mathrm{C} \mathrm{NMR}\left(75.5 \mathrm{MHz}, \mathrm{CDCl}_{3}\right): \delta 20.6,21.3,45.2,62.8,64.1$, 126.5, 127.3, 127.5, 128.5, 128.86, 128.94, 135.3, 139.1, 168.2. MS (ESI) $m / z: 266\left(\mathrm{MH}^{+}\right)$; HRMS Cacld. for $\mathrm{C}_{18} \mathrm{H}_{20} \mathrm{NO}\left(\mathrm{MH}^{+}\right)$266.1545; Found 266.1543.

( \pm )-trans-1-Isopropyl-3-phenoxy-4-phenylazetidin-2-one (12c)<smiles>CCPN1C(=O)[C@H](O)[C@@H]1c1ccccc1</smiles>

Colorless crystals, m.p. $120.5-122^{\circ} \mathrm{C}$; IR $(\mathrm{KBr})$ v $\left(\mathrm{cm}^{-1}\right)$ : $1764(\mathrm{C}=\mathrm{O}) ;{ }^{1} \mathrm{H}$ NMR $(300 \mathrm{MHz}$, $\left.\mathrm{CDCl}_{3}\right): \delta 1.06(\mathrm{~d}, J=6.6 \mathrm{~Hz}, 3 \mathrm{H}), 1.32(\mathrm{~d}, J=6.9 \mathrm{~Hz}, 3 \mathrm{H}), 3.77(\mathrm{qq}, J=6.6,6.9 \mathrm{~Hz}, 1 \mathrm{H}), 4.53(\mathrm{~d}$, $J=1.5 \mathrm{~Hz}, 1 \mathrm{H}), 4.94(\mathrm{~d}, J=1.5 \mathrm{~Hz}, 1 \mathrm{H}), 6.75-7.22(\mathrm{~m}, 5 \mathrm{H}), 7.37-7.47(\mathrm{~m}, 5 \mathrm{H}) ;{ }^{13} \mathrm{C}$ NMR $(75.5$ $\left.\mathrm{MHz}, \mathrm{CDCl}_{3}\right): \delta 20.0,21.0,45.2,62.6,86.4,115.2,121.8,126.9,128.97,129.00,129.4,137.0$, 157.0, 165.1. MS (ESI) $m / z: 282\left(\mathrm{MH}^{+}\right)$. Anal. Cacld. for $\mathrm{C}_{18} \mathrm{H}_{19} \mathrm{NO}_{2}$ (281.35): C, 76.84; H, 6.81; N, 4.98. Found: C, 76.59; H, 6.88; N, 4.81\%.

( \pm -cis-3-(1,3-Dioxoisoindolin-2-yl)-1-isopropyl-4-phenylazetidin-2-one (13c)<smiles>CCPN1C(=O)[C@H](N2C(=O)c3ccccc3C2=O)[C@@H]1c1ccccc1</smiles>

Colorless crystals, m.p. $214-215.5{ }^{\circ} \mathrm{C}$; IR $(\mathrm{KBr}) v\left(\mathrm{~cm}^{-1}\right): 1759(\mathrm{C}=\mathrm{O}), 1721(\mathrm{C}=\mathrm{O}) ;{ }^{1} \mathrm{H}$ NMR $\left(300 \mathrm{MHz}, \mathrm{CDCl}_{3}\right): \delta 1.28(\mathrm{~d}, J=6.6 \mathrm{~Hz}, 3 \mathrm{H}), 1.44$ (d, $\left.J=6.6 \mathrm{~Hz}, 3 \mathrm{H}\right), 4.00$ (heptet, $J=6.6 \mathrm{~Hz}$, $1 \mathrm{H}), 5.08(\mathrm{~d}, J=5.3 \mathrm{~Hz}, 1 \mathrm{H}), 5.42(\mathrm{~d}, J=5.3 \mathrm{~Hz}, 1 \mathrm{H}), 7.09-7.34(\mathrm{~m}, 5 \mathrm{H}), 7.59-7.68(\mathrm{~m}, 4 \mathrm{H}) ;{ }^{13} \mathrm{C}$ 
$\operatorname{NMR}\left(75.5 \mathrm{MHz}, \mathrm{CDCl}_{3}\right): \delta 20.1,21.2,45.9,59.1,60.3,123.2,127.4,128.2,128.3,131.2,134.0$, 163.4, 166.8. MS (ESI) $m / z$ : $335\left(\mathrm{MH}^{+}\right)$; Anal. Cacld. for $\mathrm{C}_{20} \mathrm{H}_{18} \mathrm{~N}_{2} \mathrm{O}_{3}$ (344.37): C, 71.84; H, 5.43; N, 8.38. Found: C, 72.04; H, 5.78; N, 8.16\%.

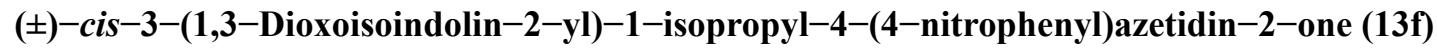<smiles>CCPN1C(=O)[C@H](N2C(=O)c3ccccc3C2=O)[C@@H]1c1ccc([N+](=O)[O-])cc1</smiles>

Colorless crystals, m.p. $238-239{ }^{\circ} \mathrm{C}$; IR $(\mathrm{KBr}) v\left(\mathrm{~cm}^{-1}\right): 1762(\mathrm{C}=\mathrm{O}), 1722(\mathrm{C}=\mathrm{O}) ;{ }^{1} \mathrm{H}$ NMR $\left(300 \mathrm{MHz}, \mathrm{CDCl}_{3}\right): \delta 1.29$ (d, $\left.J=6.6 \mathrm{~Hz}, 3 \mathrm{H}\right), 1.44(\mathrm{~d}, J=6.6 \mathrm{~Hz}, 3 \mathrm{H}), 4.03$ (heptet, $J=6.6 \mathrm{~Hz}$, $1 \mathrm{H}), 5.16(\mathrm{~d}, J=5.4 \mathrm{~Hz}, 1 \mathrm{H}), 5.51(\mathrm{~d}, J=5.4 \mathrm{~Hz}, 1 \mathrm{H}), 7.56(\mathrm{~d}, J=8.7 \mathrm{~Hz}, 2 \mathrm{H}), 7.64-7.73(\mathrm{~m}, 4 \mathrm{H})$, $8.10(\mathrm{~d}, J=8.7 \mathrm{~Hz}, 2 \mathrm{H}) ;{ }^{13} \mathrm{C} \mathrm{NMR}\left(75.5 \mathrm{MHz}, \mathrm{CDCl}_{3}\right): \delta 20.2,21.3,46.2,59.0,59.7,123.62$, 123.64, 128.5, 130.9, 134.2, 134.5, 142.0, 147.8, 163.2, 166.7. MS (ESI) $m / z: 380\left(\mathrm{MH}^{+}\right)$; Anal. Cacld. for $\mathrm{C}_{20} \mathrm{H}_{17} \mathrm{~N}_{3} \mathrm{O}_{5}$ (379.37): $\mathrm{C}, 63.32 ; \mathrm{H}, 4.52 ; \mathrm{N}, 11.08$. Found: $\mathrm{C}, 63.00 ; \mathrm{H}, 4.68 ; \mathrm{N}$, $11.07 \%$.

( \pm )-trans-3-(1,3-Dioxoisoindolin-2-yl)-1-isopropyl-4-phenylazetidin-2-one (14c)<smiles>CCPN1C(=O)C(N2C(=O)c3ccccc3C2=O)[C@@H]1c1ccccc1</smiles>

Colorless crystals, m.p. 203-204.5 ${ }^{\circ} \mathrm{C}$; IR $(\mathrm{KBr}) v\left(\mathrm{~cm}^{-1}\right): 1762(\mathrm{C}=\mathrm{O}), 1720(\mathrm{C}=\mathrm{O}) ;{ }^{1} \mathrm{H}$ NMR $\left(300 \mathrm{MHz}, \mathrm{CDCl}_{3}\right): \delta 1.13(\mathrm{~d}, J=6.9 \mathrm{~Hz}, 3 \mathrm{H}), 1.42(\mathrm{~d}, J=6.6 \mathrm{~Hz}, 3 \mathrm{H}), 3.86$ (qq, $J=6.6,6.9 \mathrm{~Hz}$, $1 \mathrm{H}), 4.95(\mathrm{~d}, J=2.2 \mathrm{~Hz}, 1 \mathrm{H}), 5.13(\mathrm{~d}, J=2.2 \mathrm{~Hz}, 1 \mathrm{H}), 7.34-7.39(\mathrm{~m}, 5 \mathrm{H}), 7.74-7.88(\mathrm{~m}, 4 \mathrm{H}) . \mathrm{MS}$ (ESI) $m / z$ : $335\left(\mathrm{MH}^{+}\right)$; Anal. Cacld. for $\mathrm{C}_{20} \mathrm{H}_{18} \mathrm{~N}_{2} \mathrm{O}_{3}$ (344.37): C, 71.84; H, 5.43; N, 8.38. Found: C, $71.54 ; \mathrm{H}, 5.63 ; \mathrm{N}, 8.16 \%$.

( \pm )-trans-3-(1,3-Dioxoisoindolin-2-yl)-1-isopropyl-4-(4-nitrophenyl)azetidin-2-one (14f)<smiles>CCCN1C(=O)[C@@]2(N3C(=O)c4ccccc4C3=O)C1[C@H]2c1ccc([N+](=O)[O-])cc1</smiles>

Yellowish crystals, m.p. 200-202 ${ }^{\circ} \mathrm{C}$; IR $(\mathrm{KBr}) v\left(\mathrm{~cm}^{-1}\right): 1765(\mathrm{C}=\mathrm{O}), 1719(\mathrm{C}=\mathrm{O}) ;{ }^{1} \mathrm{H}$ NMR $\left(300 \mathrm{MHz}, \mathrm{CDCl}_{3}\right): \delta 1.16(\mathrm{~d}, J=6.9 \mathrm{~Hz}, 3 \mathrm{H}), 1.44$ (d, $\left.J=6.6 \mathrm{~Hz}, 3 \mathrm{H}\right), 3.90$ (qq, $J=6.6 \mathrm{~Hz}, 6.9$ $\mathrm{Hz}, 1 \mathrm{H}), 5.07$ (d, $J=2.4 \mathrm{~Hz}, 1 \mathrm{H}), 5.09(\mathrm{~d}, J=2.4 \mathrm{~Hz}, 1 \mathrm{H}), 7.60(\mathrm{~d}, J=8.7 \mathrm{~Hz}, 2 \mathrm{H}), 7.72-7.91(\mathrm{~m}$, $4 \mathrm{H}), 8.28(\mathrm{~d}, J=8.7 \mathrm{~Hz}, 2 \mathrm{H}) ;{ }^{13} \mathrm{C} \mathrm{NMR}\left(75.5 \mathrm{MHz}, \mathrm{CDCl}_{3}\right): \delta 20.1,20.9,46.1,58.5,62.1,123.8$,

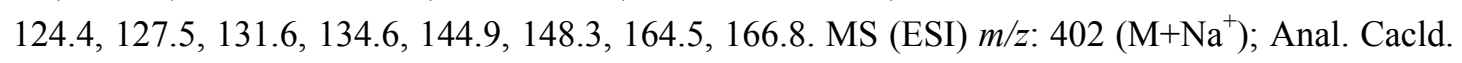
for $\mathrm{C}_{20} \mathrm{H}_{17} \mathrm{~N}_{3} \mathrm{O}_{5}$ (379.37): C, 63.32; H, 4.52; N, 11.08. Found: C, 63.00; H, 4.68; N, 11.07\%. 
( \pm - - cis $^{-3}$-Chloro-1-isopropyl-4-(4-methoxyphenyl)azetidin-2-one (15a)<smiles>CCCN1C(=O)[C@H](Cl)[C@H]1c1ccc(OC)cc1</smiles>

Yellowish crystals, m.p. $73.5-75{ }^{\circ} \mathrm{C}$; IR $(\mathrm{KBr}) v\left(\mathrm{~cm}^{-1}\right)$ : $1763(\mathrm{C}=\mathrm{O}) ;{ }^{1} \mathrm{H}$ NMR $(300 \mathrm{MHz}$, $\left.\mathrm{CDCl}_{3}\right): \delta 1.11(\mathrm{~d}, J=6.6 \mathrm{~Hz}, 3 \mathrm{H}), 1.32(\mathrm{~d}, J=6.6 \mathrm{~Hz}, 3 \mathrm{H}), 3.77$ (heptet, $\left.J=6.6 \mathrm{~Hz}, 1 \mathrm{H}\right), 3.83(\mathrm{~s}$, $3 \mathrm{H}), 4.92(\mathrm{~d}, J=4.8 \mathrm{~Hz}, 1 \mathrm{H}), 5.01(\mathrm{~d}, J=4.8 \mathrm{~Hz}, 1 \mathrm{H}), 6.94(\mathrm{~d}, J=8.7 \mathrm{~Hz}, 2 \mathrm{H}), 7.26(\mathrm{~d}, J=8.7$ $\mathrm{Hz}, 2 \mathrm{H}) ;{ }^{13} \mathrm{C}$ NMR $\left(75.5 \mathrm{MHz}, \mathrm{CDCl}_{3}\right): \delta 20.2,21.1,45.8,55.2,59.6,60.4,113.7,126.0,129.7$, 160.2, 163.5. MS (ESI) $m / z: 254\left(\mathrm{MH}^{+}\right)$; Anal. Cacld. for $\mathrm{C}_{13} \mathrm{H}_{16} \mathrm{ClNO}_{2}$ (253.72): C, 61.54; $\mathrm{H}$, 6.36 ; N, 5.52. Found: C, 61.27; H, 6.48; N, 5.71\%.

( \pm )-cis-3-Chloro-1-isopropyl-4-(4-nitrophenyl)azetidin-2-one (15f)<smiles>NC(=O)[C@H]1C(=O)[C@H](Cl)[C@@H]1c1ccc([N+](=O)[O-])cc1</smiles>

Yellowish crystals, m.p. $102-103{ }^{\circ} \mathrm{C}$; IR $(\mathrm{KBr}) v\left(\mathrm{~cm}^{-1}\right): 1768(\mathrm{C}=\mathrm{O}) ;{ }^{1} \mathrm{H}$ NMR $(300 \mathrm{MHz}$, $\left.\mathrm{CDCl}_{3}\right): \delta 1.14(\mathrm{~d}, J=6.6 \mathrm{~Hz}, 3 \mathrm{H}), 1.34(\mathrm{~d}, J=6.6 \mathrm{~Hz}, 3 \mathrm{H}), 3.83$ (heptet, $\left.J=6.6 \mathrm{~Hz}, 1 \mathrm{H}\right), 5.07$ (d, $J=5.1 \mathrm{~Hz}, 1 \mathrm{H}), 5.10(\mathrm{~d}, J=5.1 \mathrm{~Hz}, 1 \mathrm{H}), 7.53(\mathrm{~d}, J=8.7 \mathrm{~Hz}, 2 \mathrm{H}), 8.29(\mathrm{~d}, J=8.7 \mathrm{~Hz}, 2 \mathrm{H}) ;{ }^{13} \mathrm{C}$ NMR (75.5 MHz, $\mathrm{CDCl}_{3}$ ): $\delta 20.3,21.1,46.3,59.1,59.8,123.6,129.2,141.9,148.4,163.4 . \mathrm{MS}$ (ESI) $m / z$ : $269\left(\mathrm{MH}^{+}\right)$; Anal. Cacld. for $\mathrm{C}_{12} \mathrm{H}_{13} \mathrm{ClN}_{2} \mathrm{O}_{3}$ (268.70): C, 53.64; H, 4.88; N, 10.43 . Found: C, 53.47; H, 4.68; N, 10.70\%.

( \pm )-trans-3-Chloro-1-isopropyl-4-(4-methoxyphenyl)azetidin-2-one (16a)<smiles>CCCN1C(=O)[C@@H](Cl)[C@H]1c1ccc(OC)cc1</smiles>

Yellowish oil; IR $(\mathrm{KBr}) v\left(\mathrm{~cm}^{-1}\right): 1767(\mathrm{C}=\mathrm{O}) ;{ }^{1} \mathrm{H}$ NMR (300 MHz, $\left.\mathrm{CDCl}_{3}\right): \delta 1.06(\mathrm{~d}, J=6.6$ $\mathrm{Hz}, 3 \mathrm{H}), 1.30$ (d, $J=6.6 \mathrm{~Hz}, 3 \mathrm{H}), 3.73$ (heptet, $J=6.6 \mathrm{~Hz}, 1 \mathrm{H}), 3.83$ (s, $3 \mathrm{H}), 4.44$ (d, $J=1.8 \mathrm{~Hz}$, $1 \mathrm{H}), 4.48(\mathrm{~d}, J=1.8 \mathrm{~Hz}, 1 \mathrm{H}), 6.92-6.95(\mathrm{~m}, 2 \mathrm{H}), 7.27-7.29(\mathrm{~m}, 2 \mathrm{H}) ;{ }^{13} \mathrm{C}$ NMR $(75.5 \mathrm{MHz}$, $\left.\mathrm{CDCl}_{3}\right): \delta 20.0,21.0,45.7,55.3,62.5,64.7,114.4,127.9,128.3,160.3,163.5$. MS (ESI) $m / z: 254$ $\left(\mathrm{MH}^{+}\right)$; HRMS Cacld. for $\mathrm{C}_{13} \mathrm{H}_{17} \mathrm{ClNO}_{2}\left(\mathrm{MH}^{+}\right)$254.0948; Found 254.0942.

( \pm --trans-3-Chloro-1-isopropyl-4-(4-nitrophenyl)azetidin-2-one (16f)<smiles>O=C1[C@H](Cl)C(c2ccc([N+](=O)[O-])cc2)[C@H]1Cl</smiles> 
Yellow oil; IR $(\mathrm{KBr}) v\left(\mathrm{~cm}^{-1}\right): 1769(\mathrm{C}=\mathrm{O}) ;{ }^{1} \mathrm{H}$ NMR $\left(300 \mathrm{MHz}, \mathrm{CDCl}_{3}\right): \delta 1.09(\mathrm{~d}, J=6.6 \mathrm{~Hz}$, $3 \mathrm{H}), 1.33(\mathrm{~d}, J=6.6 \mathrm{~Hz}, 3 \mathrm{H}), 3.80$ (heptet, $J=6.6 \mathrm{~Hz}, 1 \mathrm{H}), 4.45$ (d, $J=1.8 \mathrm{~Hz}, 1 \mathrm{H}), 4.63$ (d, $J=$ $1.8 \mathrm{~Hz}, 1 \mathrm{H}), 7.58(\mathrm{~d}, J=8.7 \mathrm{~Hz}, 2 \mathrm{H}), 8.30(\mathrm{~d}, J=8.7 \mathrm{~Hz}, 2 \mathrm{H})$. MS (ESI) $m / z: 269\left(\mathrm{MH}^{+}\right) ; \mathrm{HRMS}$ Cacld. for $\mathrm{C}_{12} \mathrm{H}_{14} \mathrm{ClN}_{2} \mathrm{O}_{3}\left(\mathrm{MH}^{+}\right)$269.0693; Found 269.0697 . 
( \pm )-cis-1-Isopropyl-3-methyl-4-(4-trifluoromethylphenyl)azetidin-2-one (7e)<smiles>CCPN1C(=O)[C@H](C)[C@H]1c1ccc(C(F)(F)F)cc1</smiles>

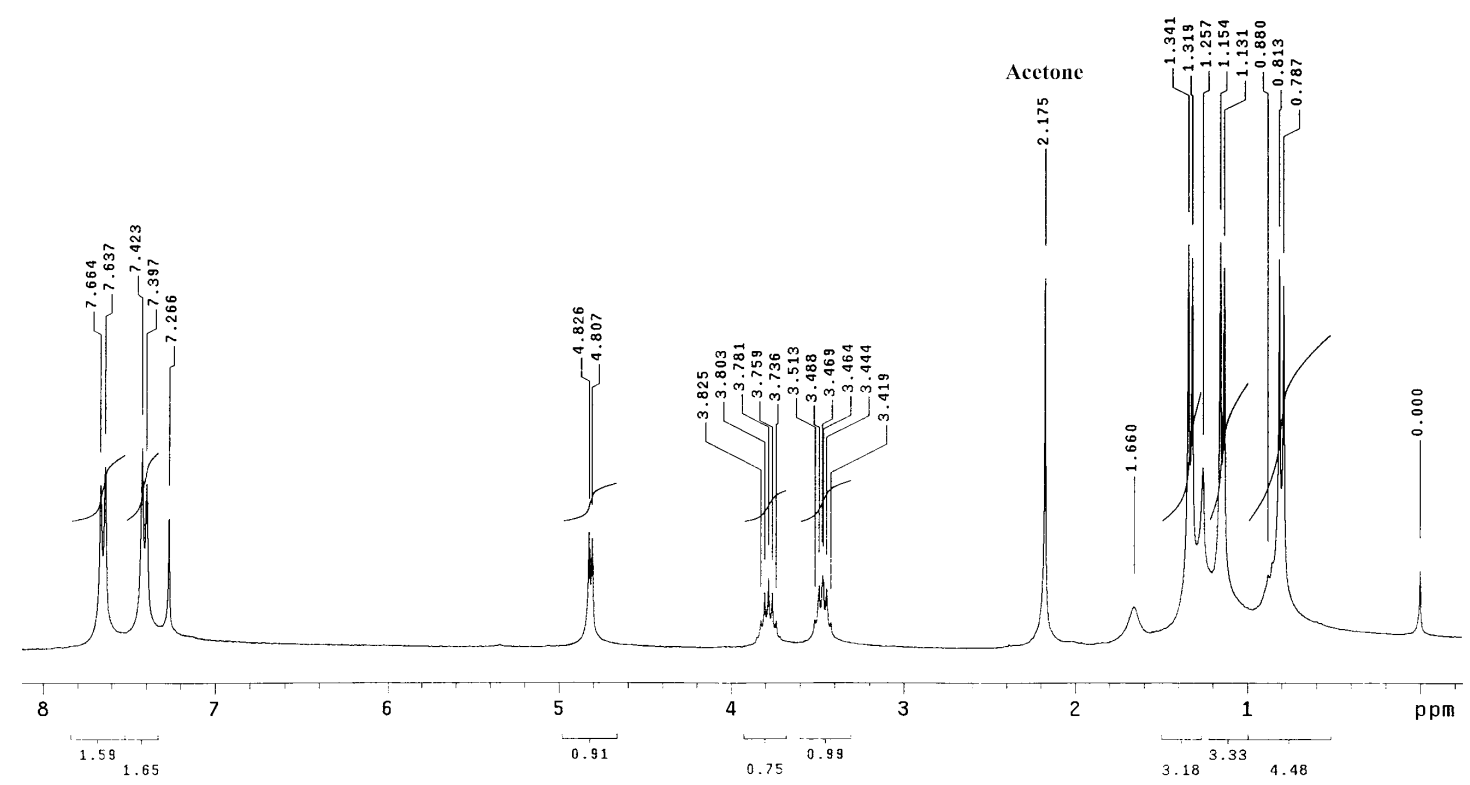


( \pm )-trans $-1-$ Isopropyl-4-(4-methoxylphenyl)-3-methylazetidin-2-one (8a)<smiles>CCCN1C(=O)[C@H](C)[C@H]1c1ccc(OC)cc1</smiles>
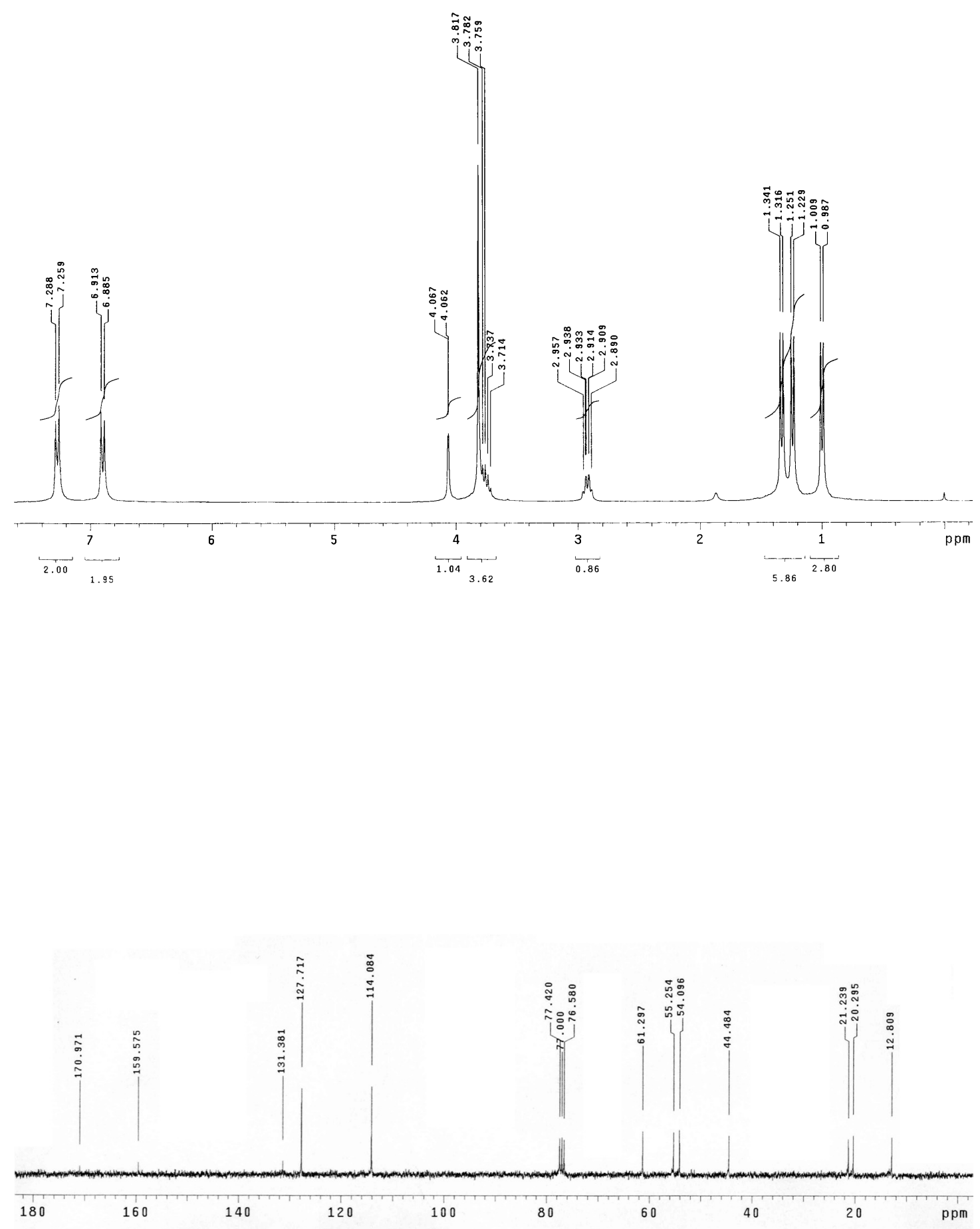
( \pm )-trans-1-Isopropyl-3-methyl-4-(4-methylphenyl)azetidin-2-one (8b)<smiles>CCCN1C(=O)[C@H](C)[C@H]1c1ccc(C)cc1</smiles>
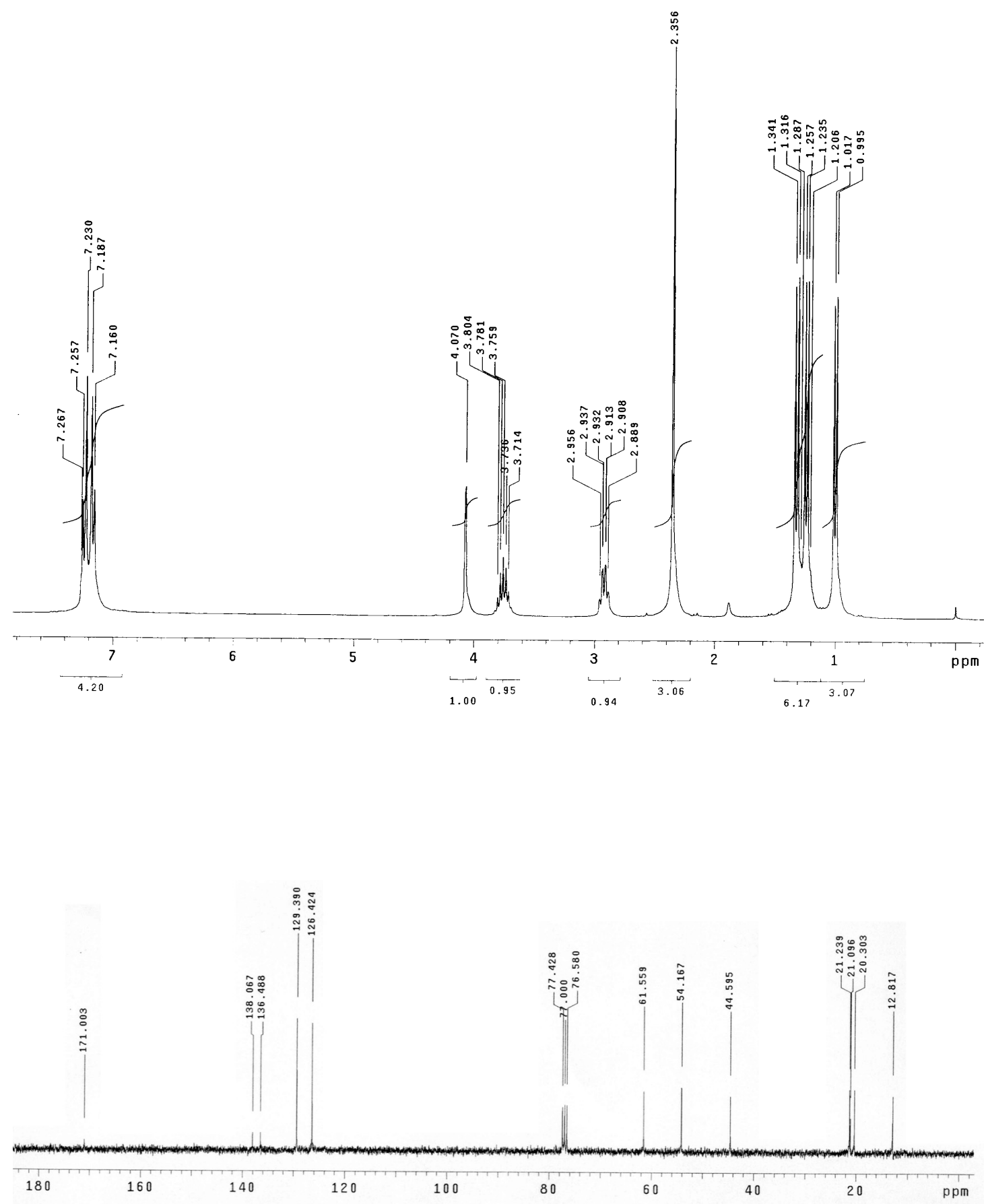
( \pm )-trans-1-Isopropyl-3-methyl-4-phenylazetidin-2-one (8c)<smiles>CCCN1C(=O)C(C)[C@H]1c1ccccc1</smiles>
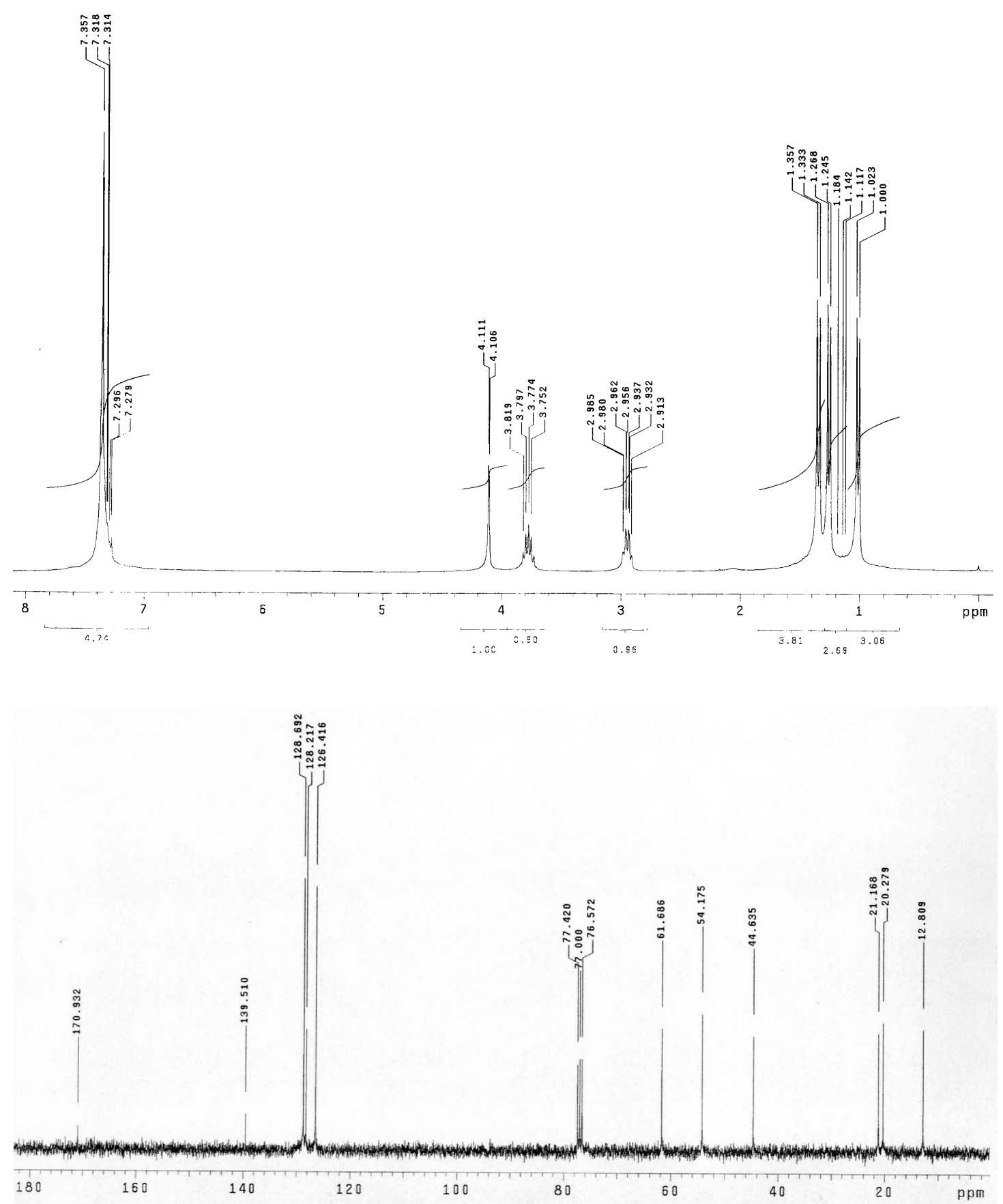
( \pm -) trans $^{-4-(4-C h l o r o p h e n y l)-1-i s o p r o p y l-3-m e t h y l a z e t i d i n-2-o n e ~(8 d) ~}$<smiles>CCCN1C(=O)[C@H](C)[C@H]1c1ccc(Cl)cc1</smiles>
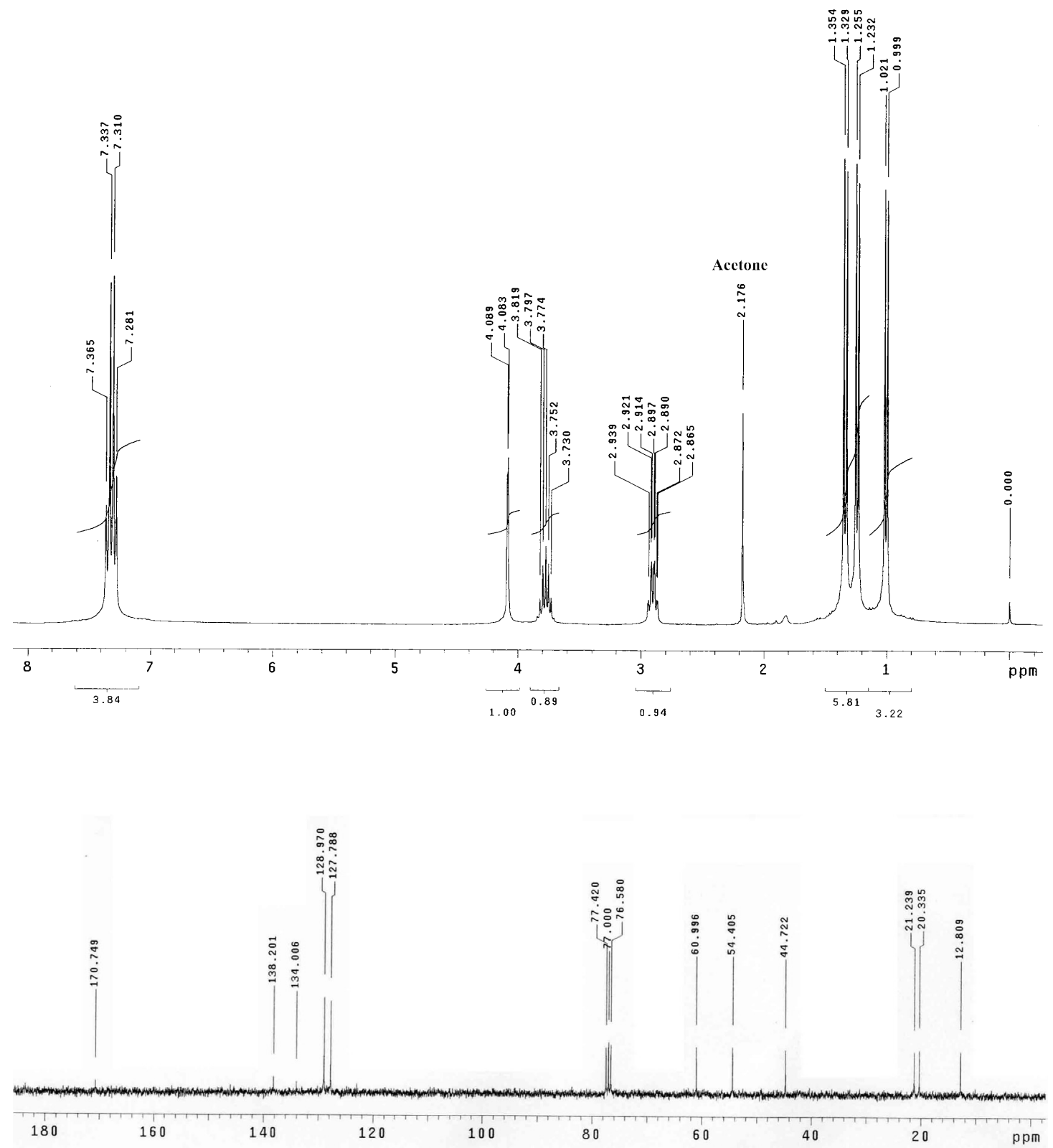
( \pm )-trans-1-Isopropyl-3-methyl-4-(4-trifluoromethylphenyl)azetidin-2-one (8e)<smiles>CCCN1C(=O)[C@@H](C)[C@H]1c1ccc(C(F)(F)F)cc1</smiles>
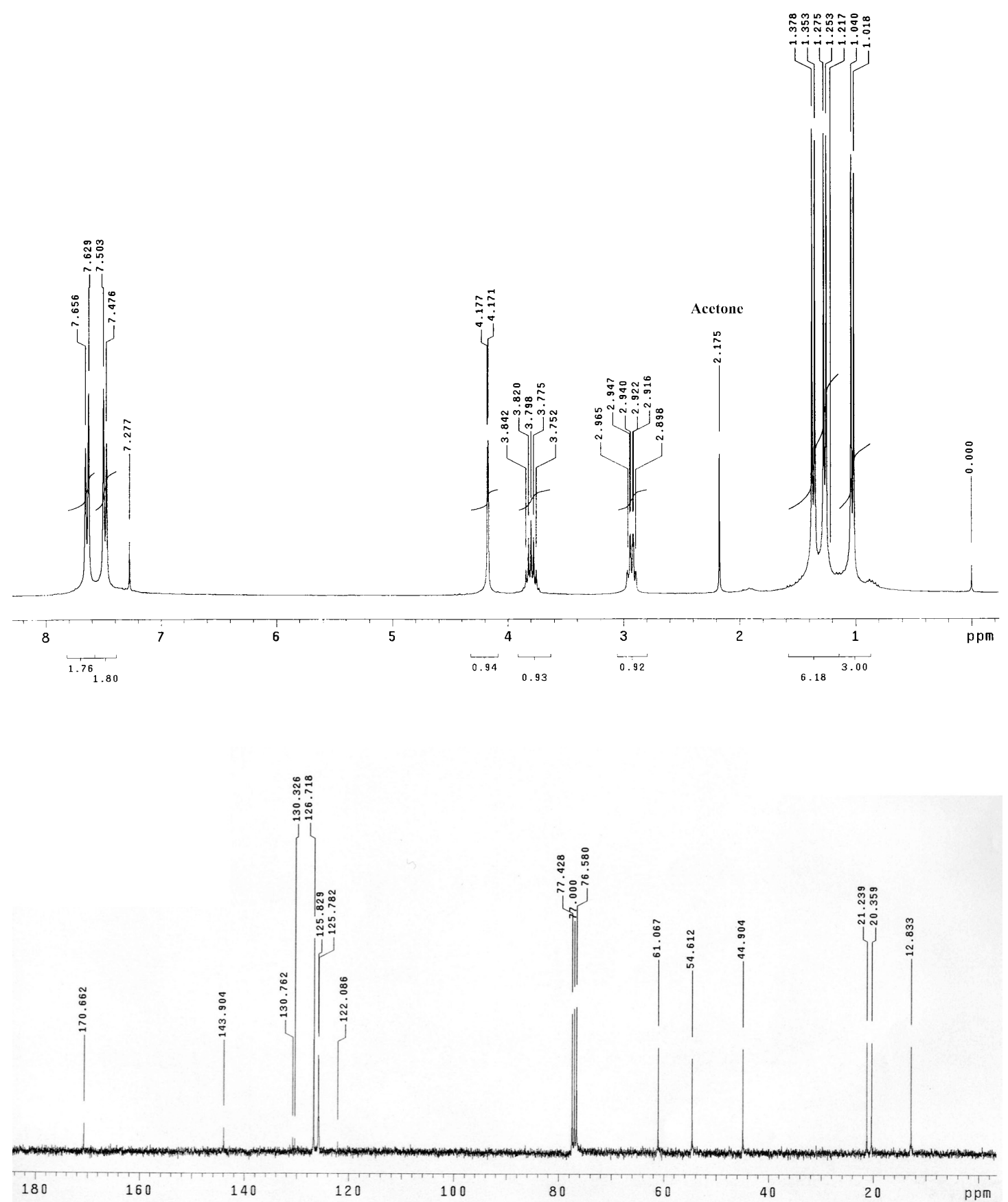
( \pm )-cis-1-Isopropyl-4-(4-methoxylphenyl)-3-phenylazetidin-2-one (9a)<smiles>CCCN1C(=O)[C@H](c2ccccc2)[C@H]1c1ccc(OC)cc1</smiles>

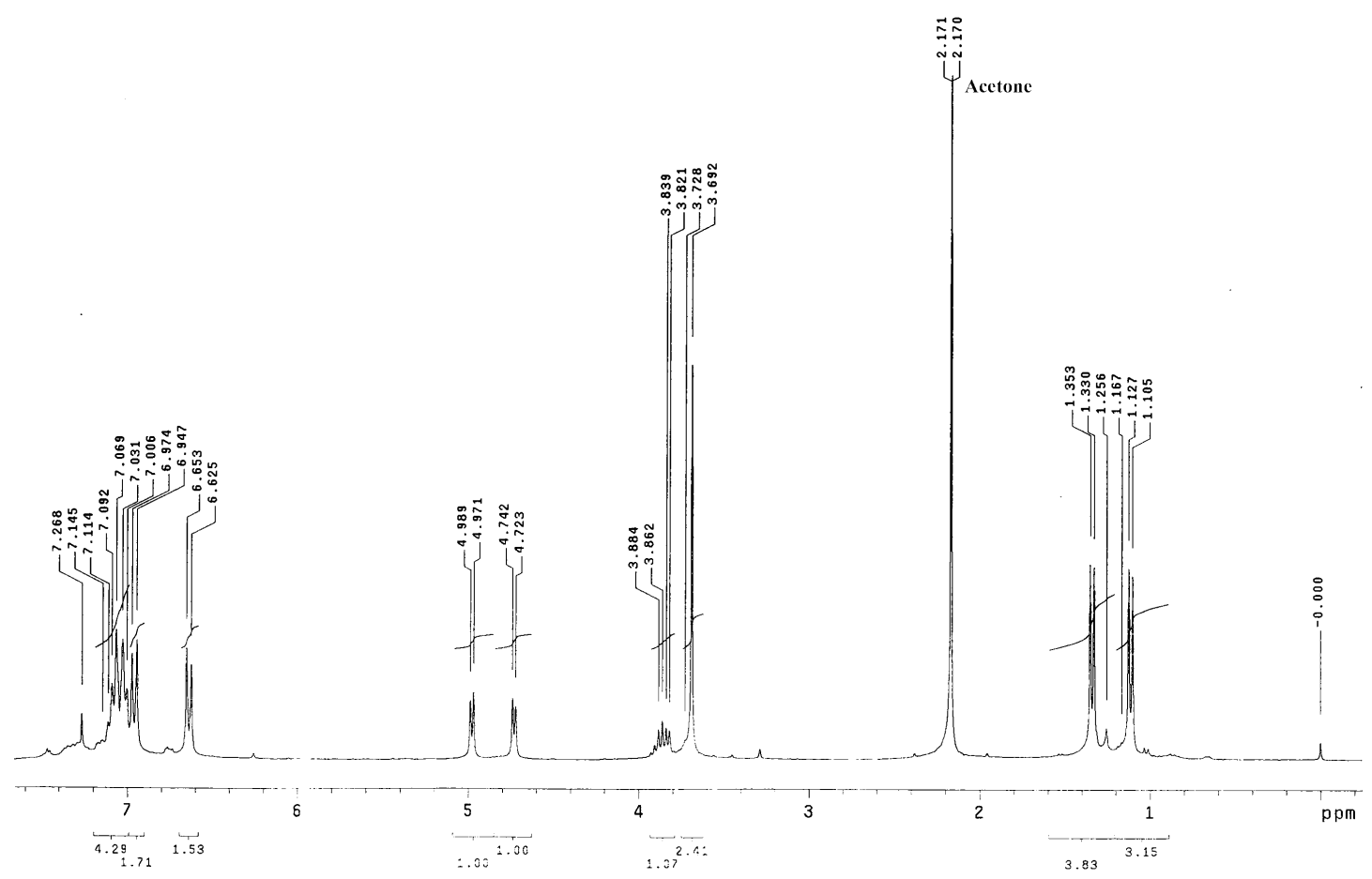


( \pm )-trans $-1-$ Isopropyl-4-(4-methoxylphenyl)-3-phenylazetidin-2-one (10a)<smiles>CCCN1C(=O)[C@@H](c2ccccc2)[C@H]1c1ccc(OC)cc1</smiles>
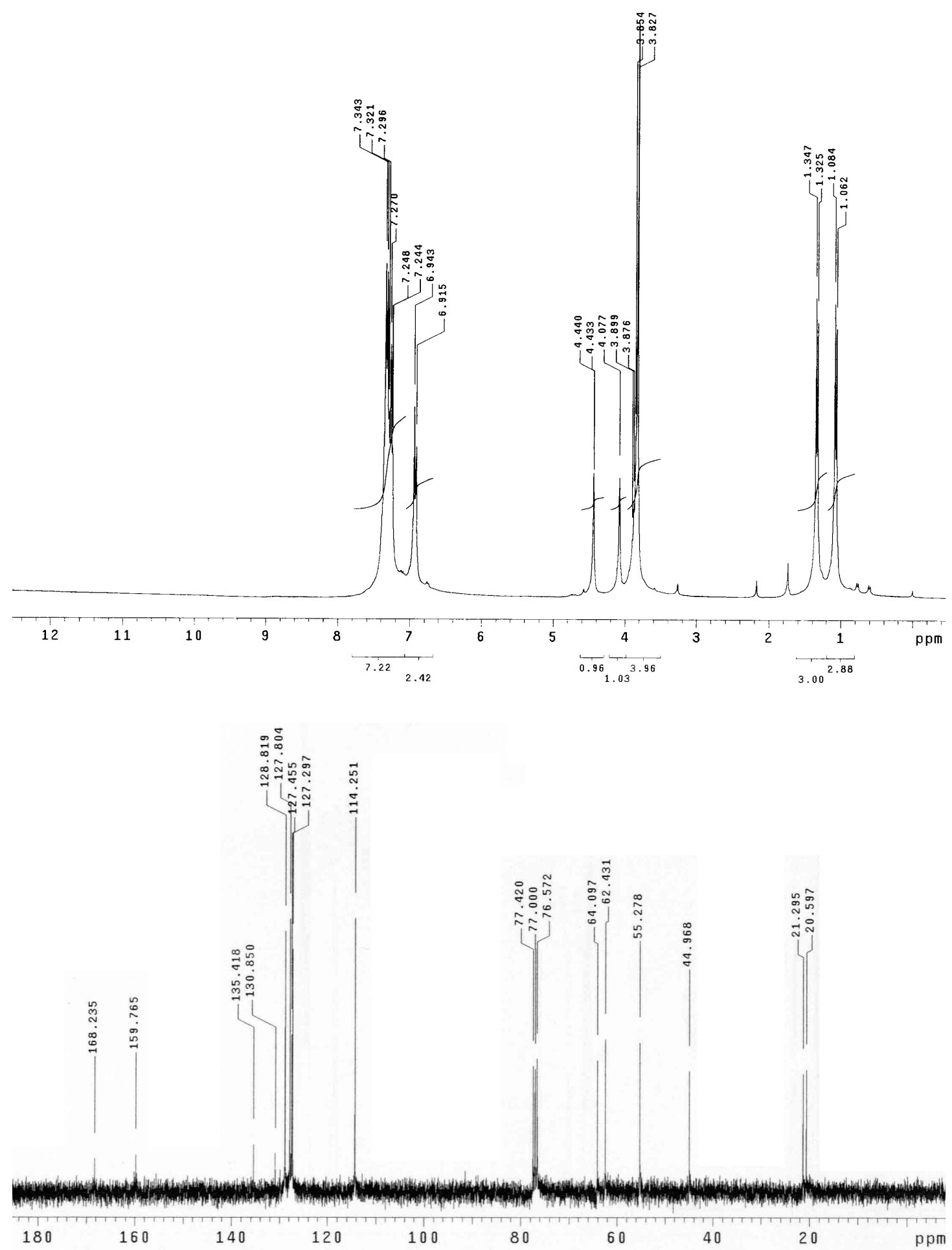
( \pm )-trans $-1-$ Isopropyl-3,4-diphenylazetidin-2-one (10c)<smiles>CCCN1C(=O)[C@@H](c2ccccc2)[C@H]1c1ccccc1</smiles>
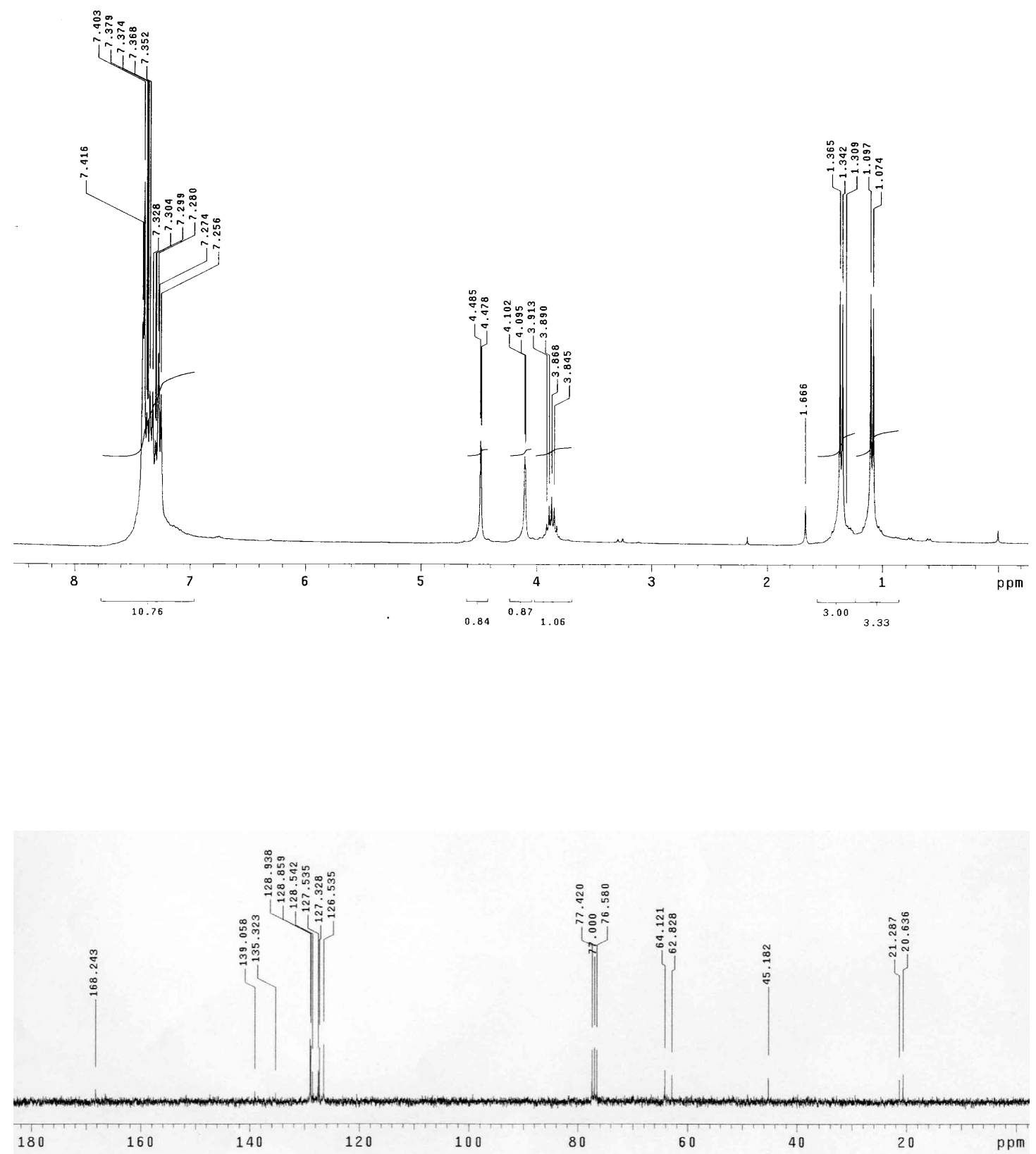
( \pm )-cis-1-Isopropyl-4-(4-nitrophenyl)-3-phenoxyazetidin-2-one (11f)<smiles>CPN1C(=O)[C@H](c2ccc([N+](=O)[O-])cc2)[C@@H]1c1ccccc1</smiles>
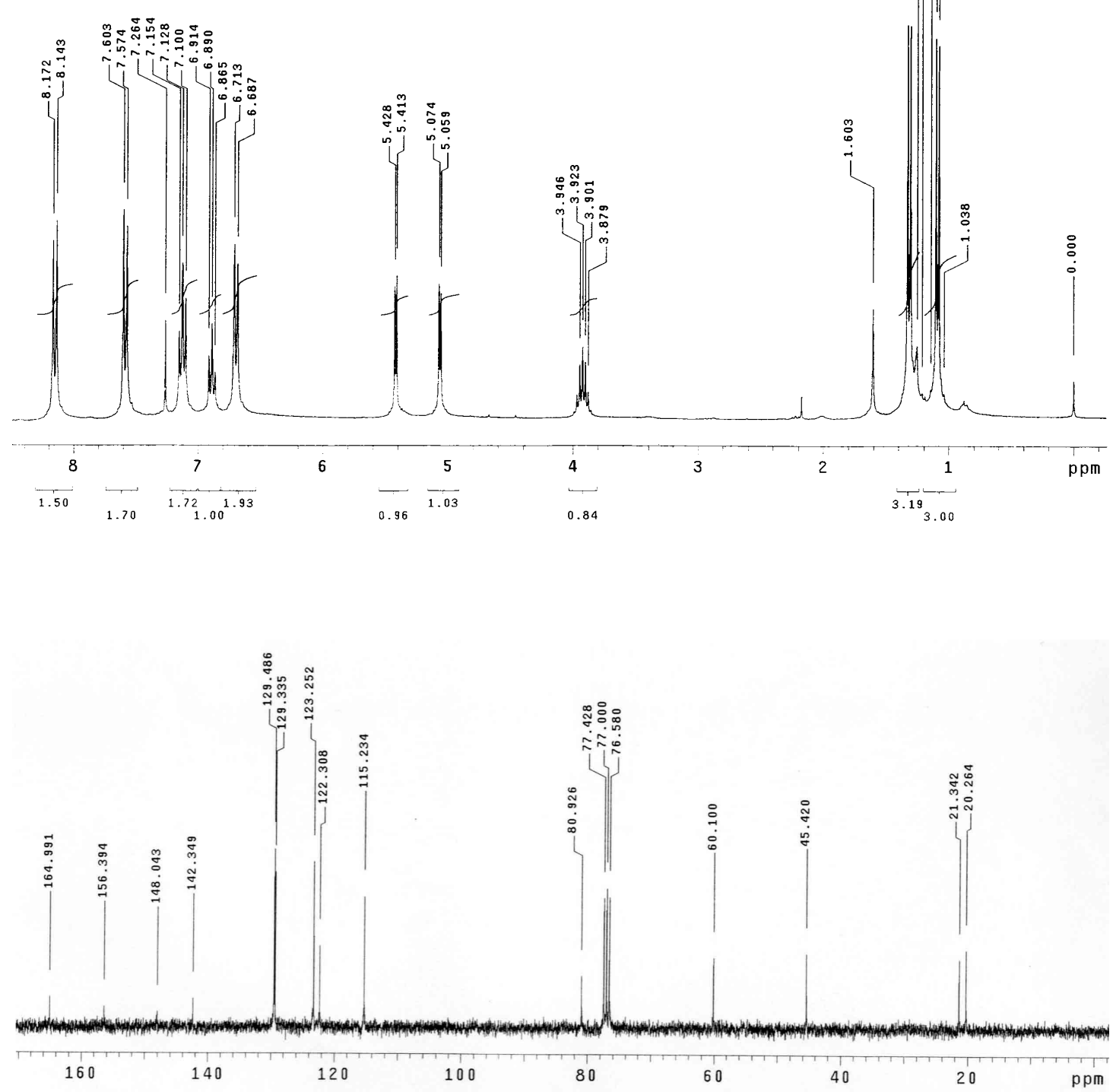
( \pm )-trans-1-Isopropyl-3-phenoxy-4-phenylazetidin-2-one (12c)<smiles>CCCN1C(=O)[C@@H](O)[C@H]1c1ccccc1</smiles>
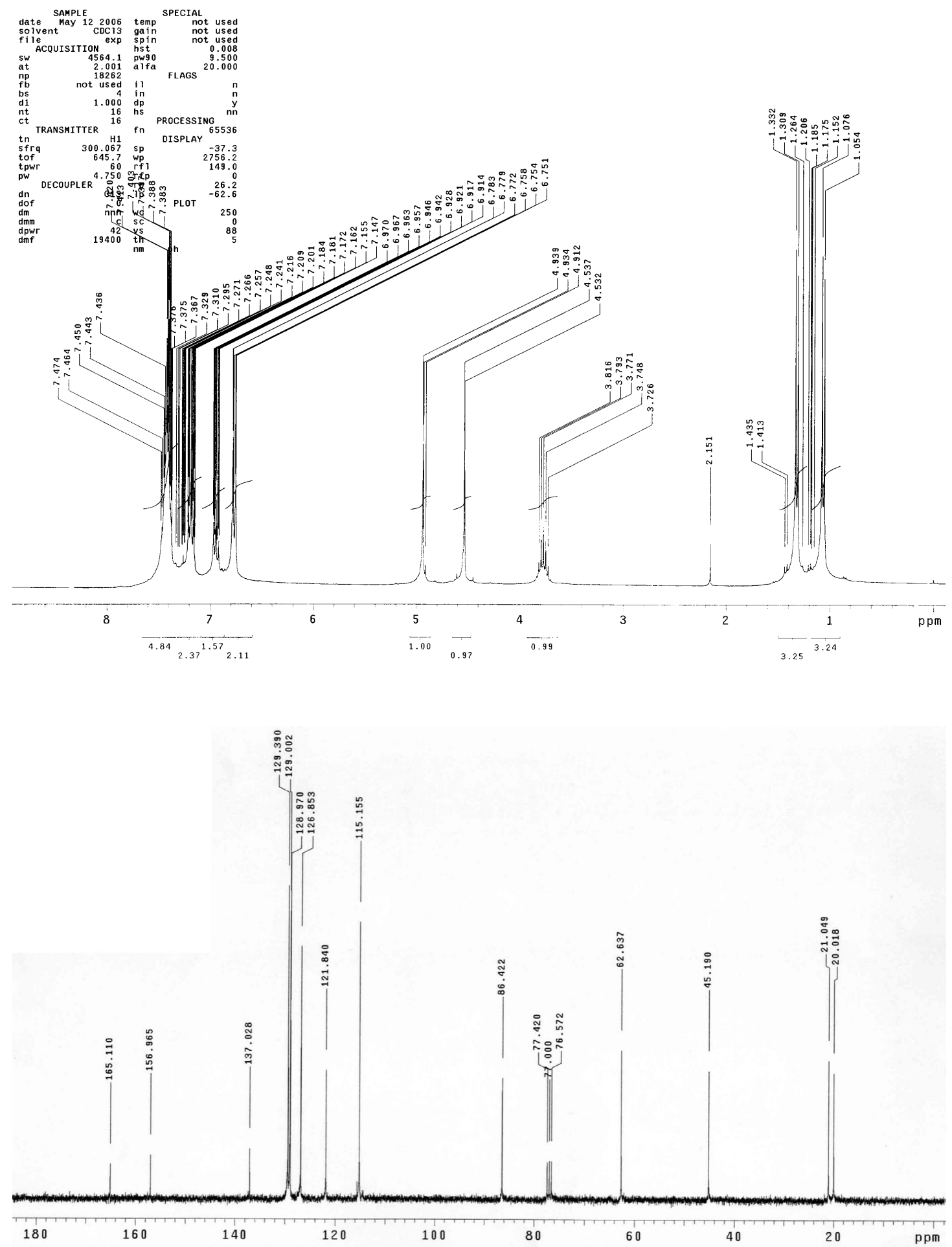
( \pm -) trans $^{-1-I s o p r o p y l-4-(4-n i t r o p h e n y l)-3-p h e n o x y a z e t i d i n-2-o n e ~(12 f) ~}$<smiles>CCPN1C(=O)[C@H](Oc2ccccc2)[C@@H]1c1ccc([N+](=O)[O-])cc1</smiles>
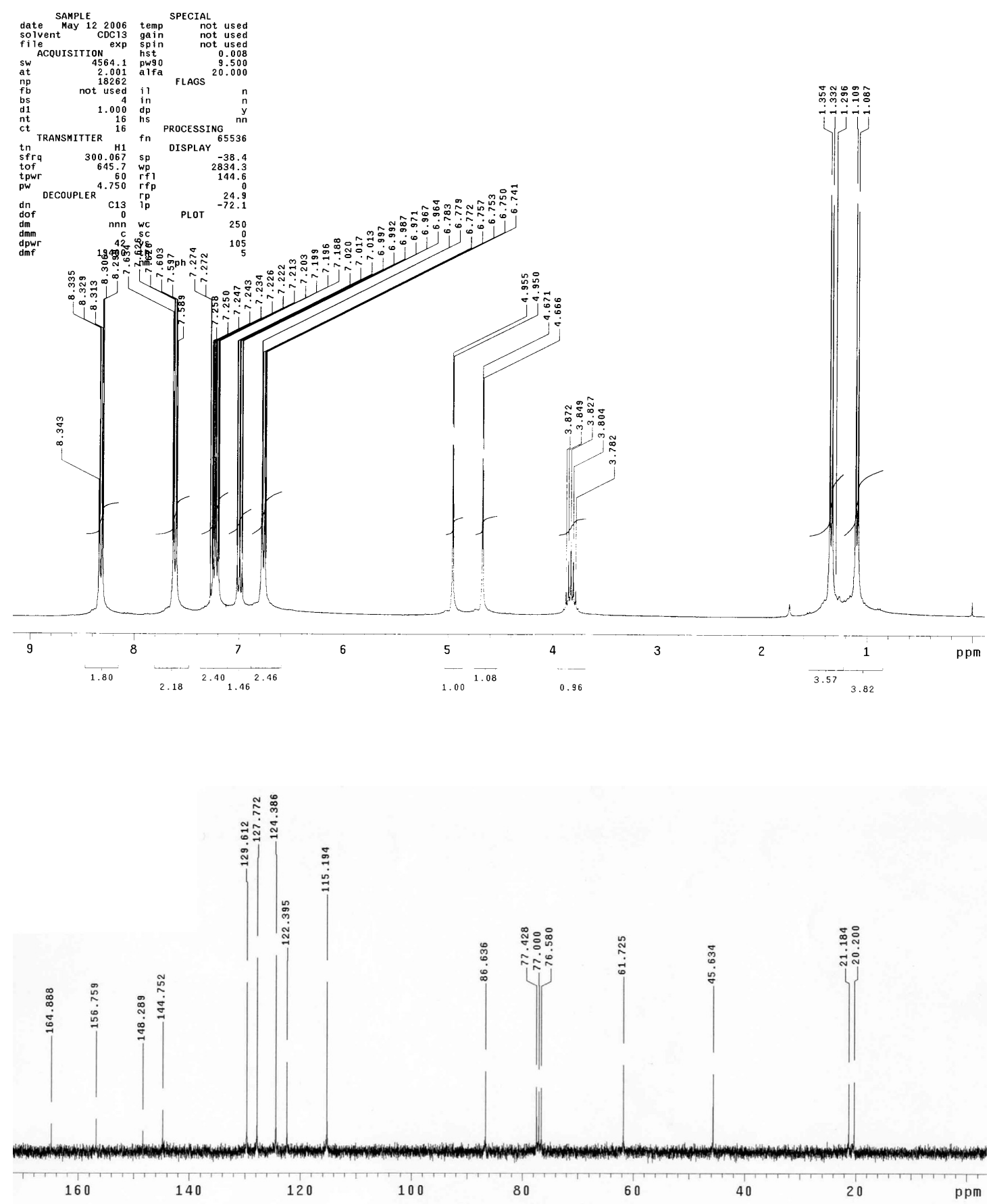
( \pm -cis-3-(1,3-Dioxoisoindolin-2-yl)-1-isopropyl-4-phenylazetidin-2-one (13c)
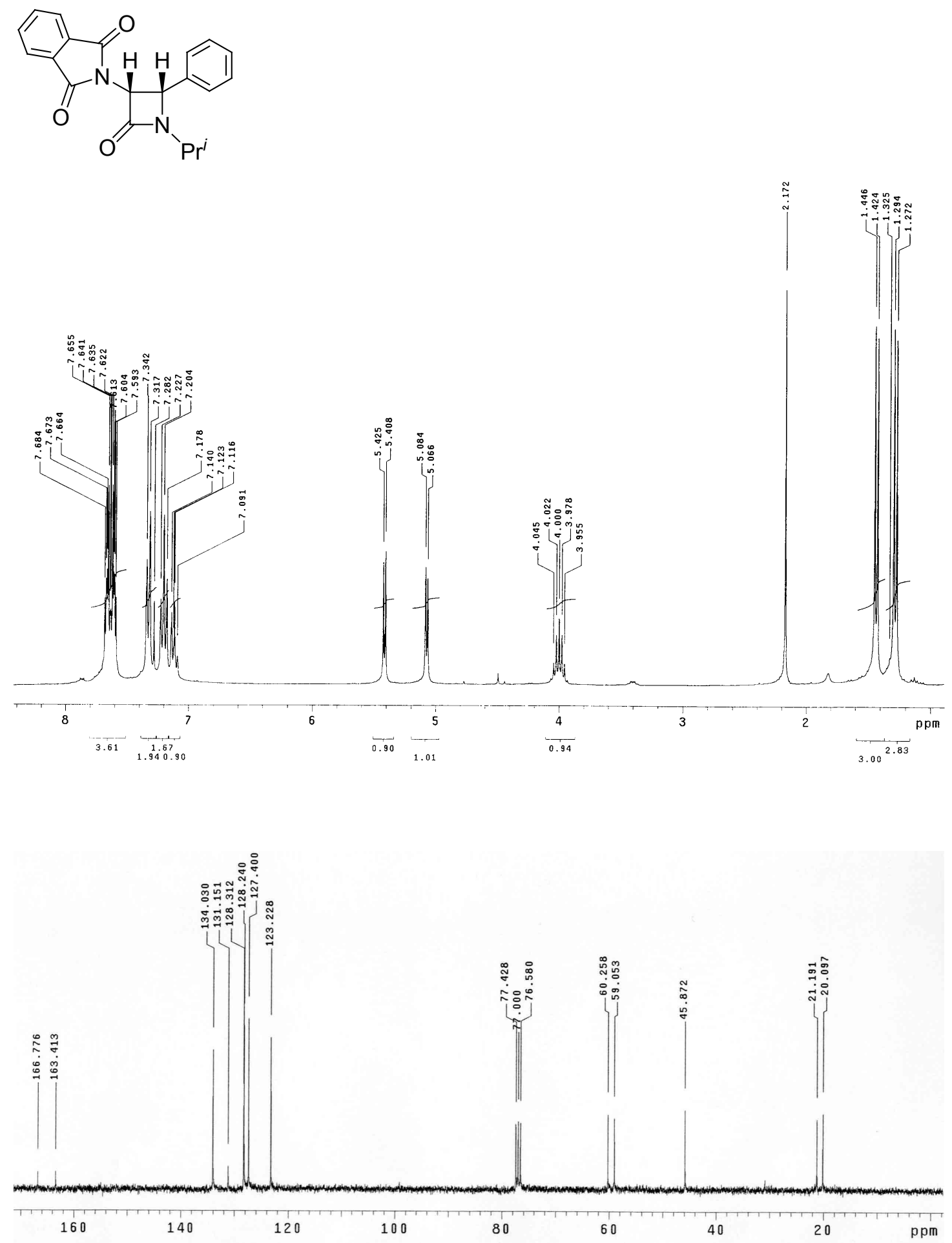
( \pm )-cis-3-(1,3-Dioxoisoindolin-2-yl)-1-isopropyl-4-(4-nitrophenyl)azetidin-2-one (13f)<smiles>CCPN1C(=O)[C@H](N2C(=O)c3ccccc3C2=O)[C@@H]1c1ccc([N+](=O)[O-])cc1</smiles>
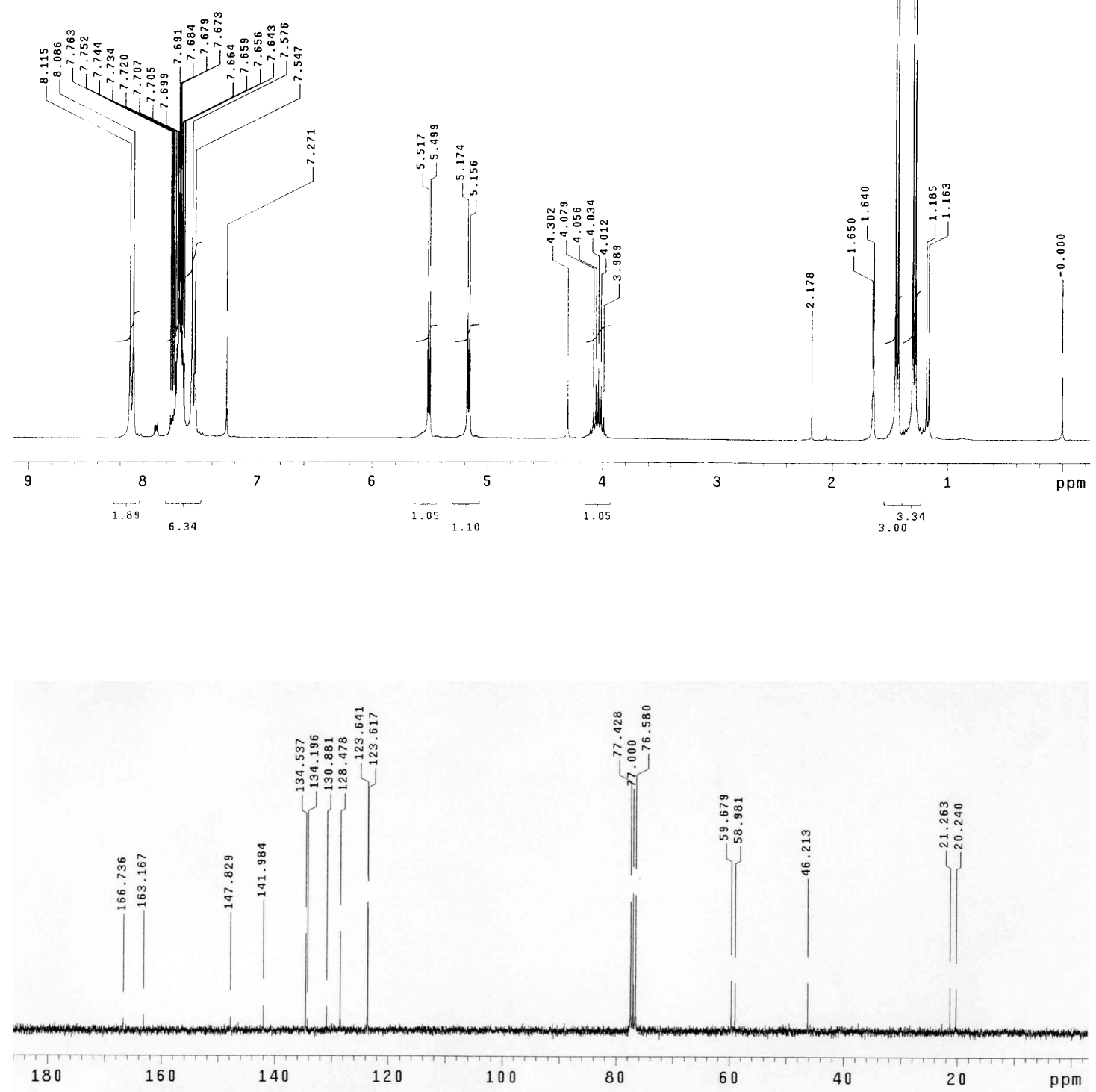
( $)$-trans-3-(1,3-Dioxoisoindolin-2-yl)-1-isopropyl-4-phenylazetidin-2-one (14c)

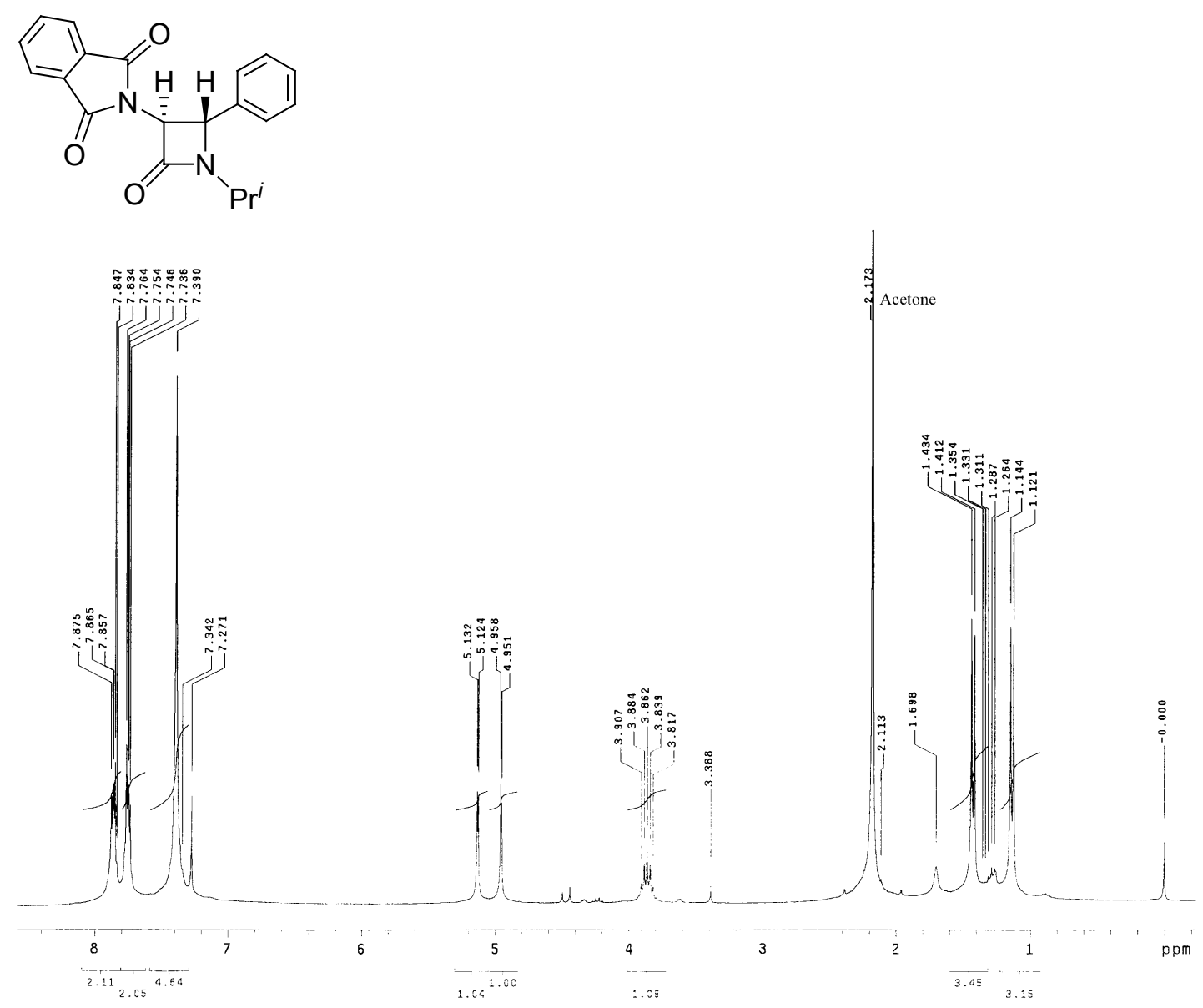


( \pm -trans-3-(1,3-Dioxoisoindolin-2-yl)-1-isopropyl-4-(4-nitrophenyl)azetidin-2-one (14f)<smiles>CCCN1C(=O)[C@@H](c2ccc([N+](=O)[O-])cc2)[C@@H]1N1C(=O)c2ccccc2C1=O</smiles>
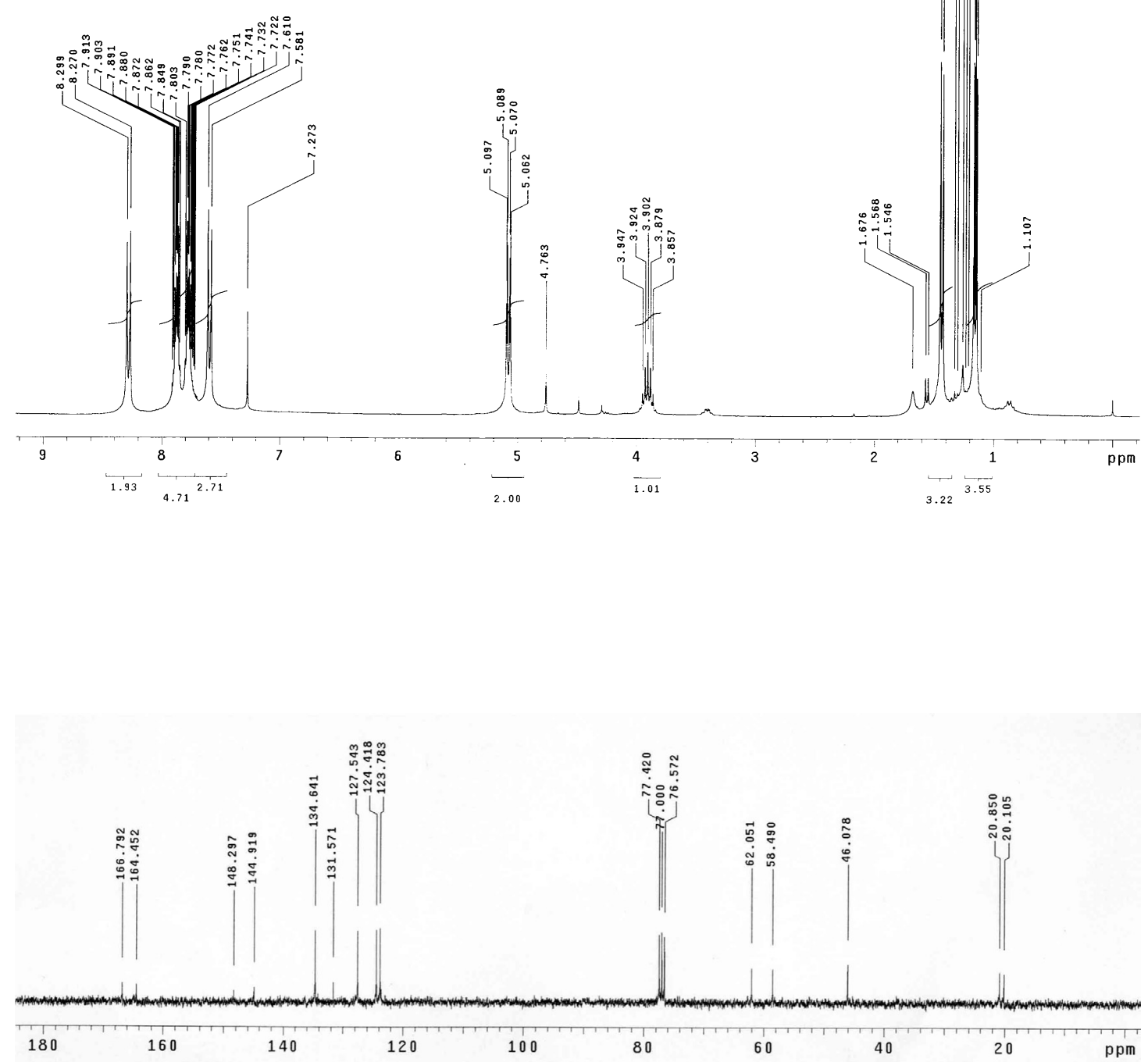
( \pm - - cis $^{-3}$-Chloro-1-isopropyl-4-(4-methoxyphenyl)azetidin-2-one (15a)<smiles>CCCN1C(=O)[C@@H](Cl)[C@H]1c1ccc(OC)cc1</smiles>
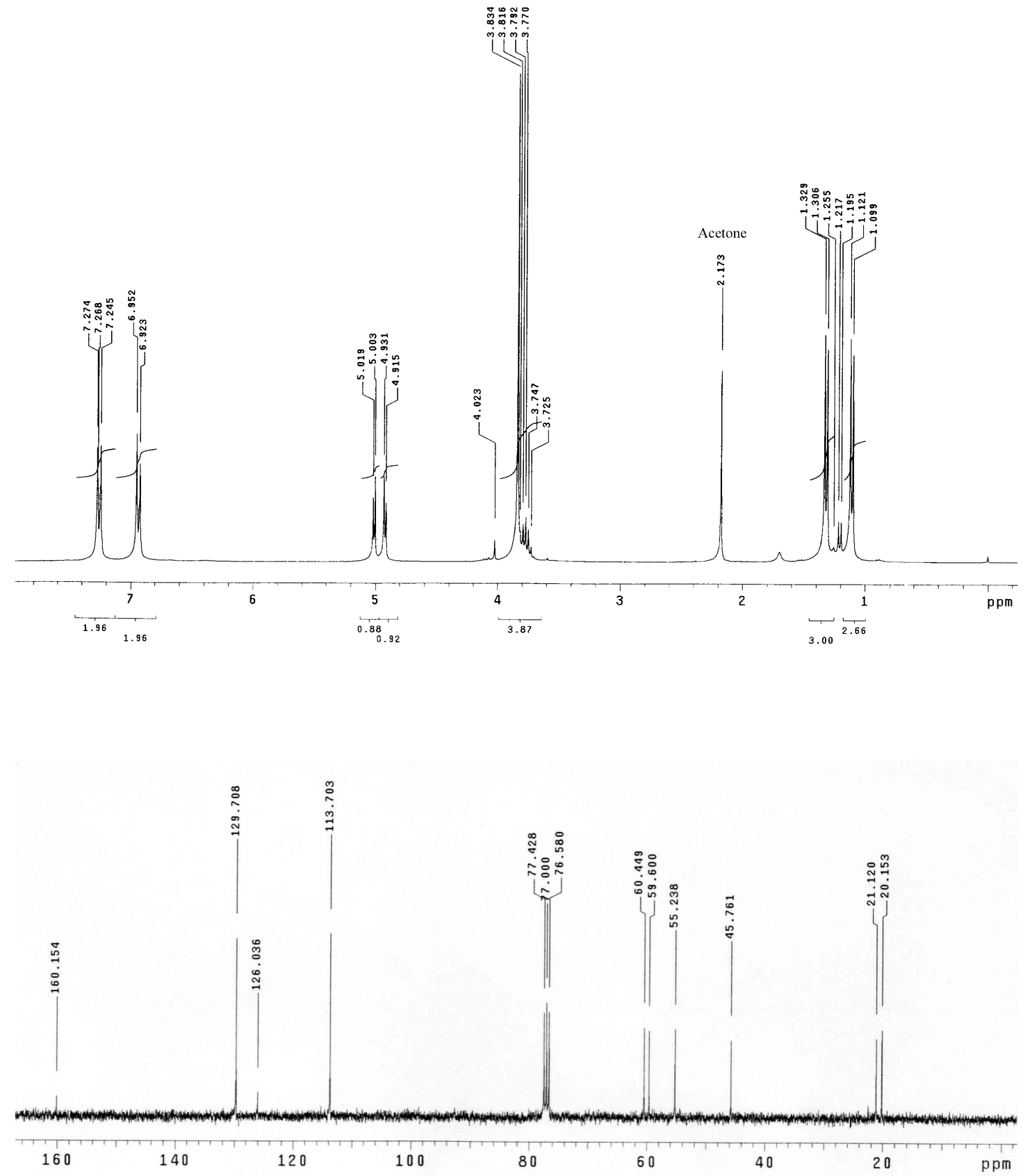


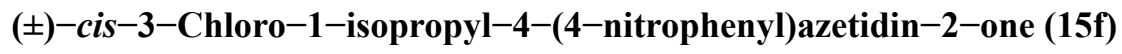<smiles>O=C1[C@H](Cl)C(c2ccc([N+](=O)[O-])cc2)[C@H]1Cl</smiles>
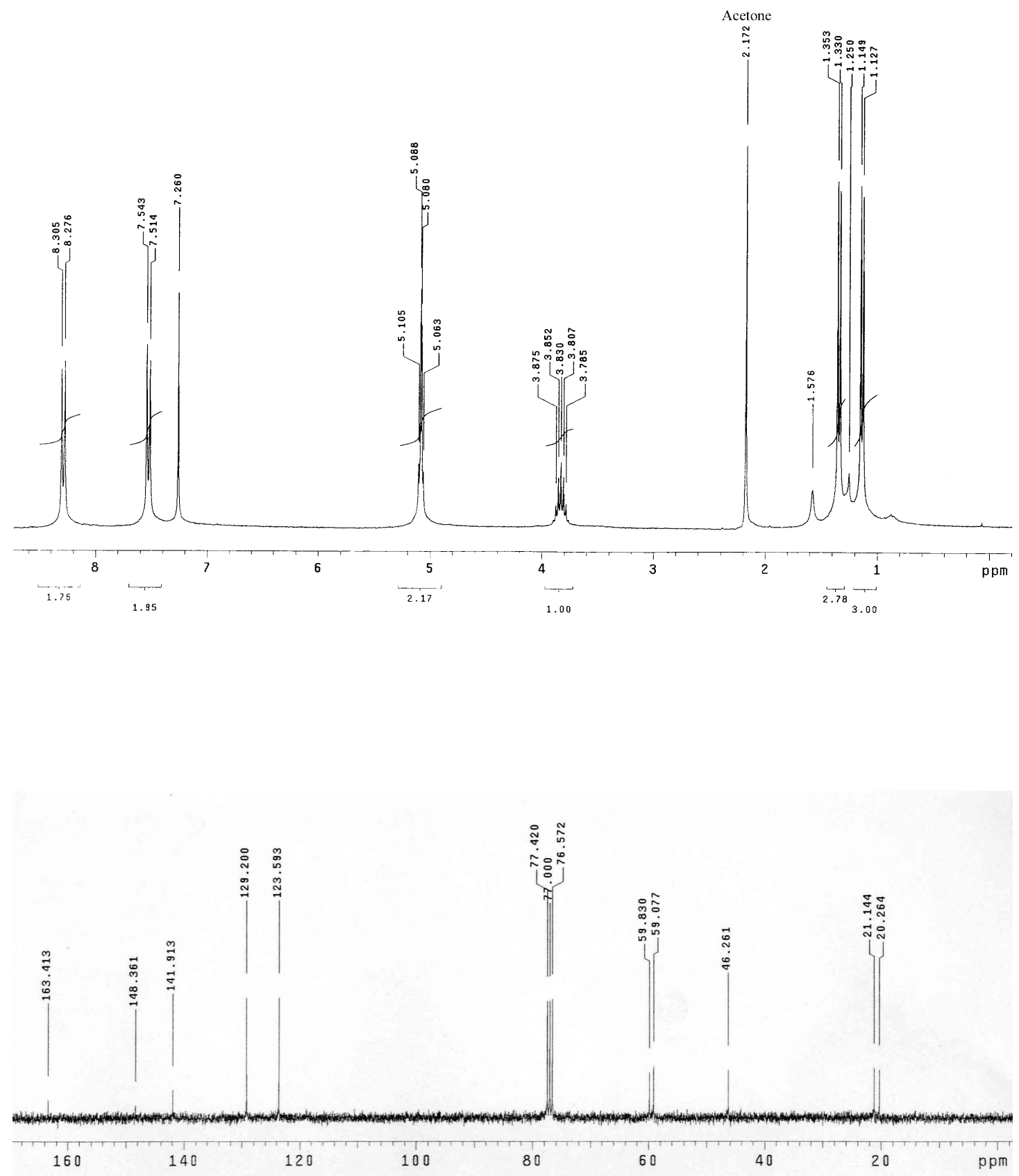


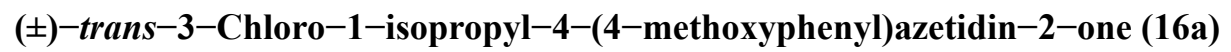<smiles>CCCN1C(=O)[C@@H](Cl)[C@H]1c1ccc(OC)cc1</smiles>
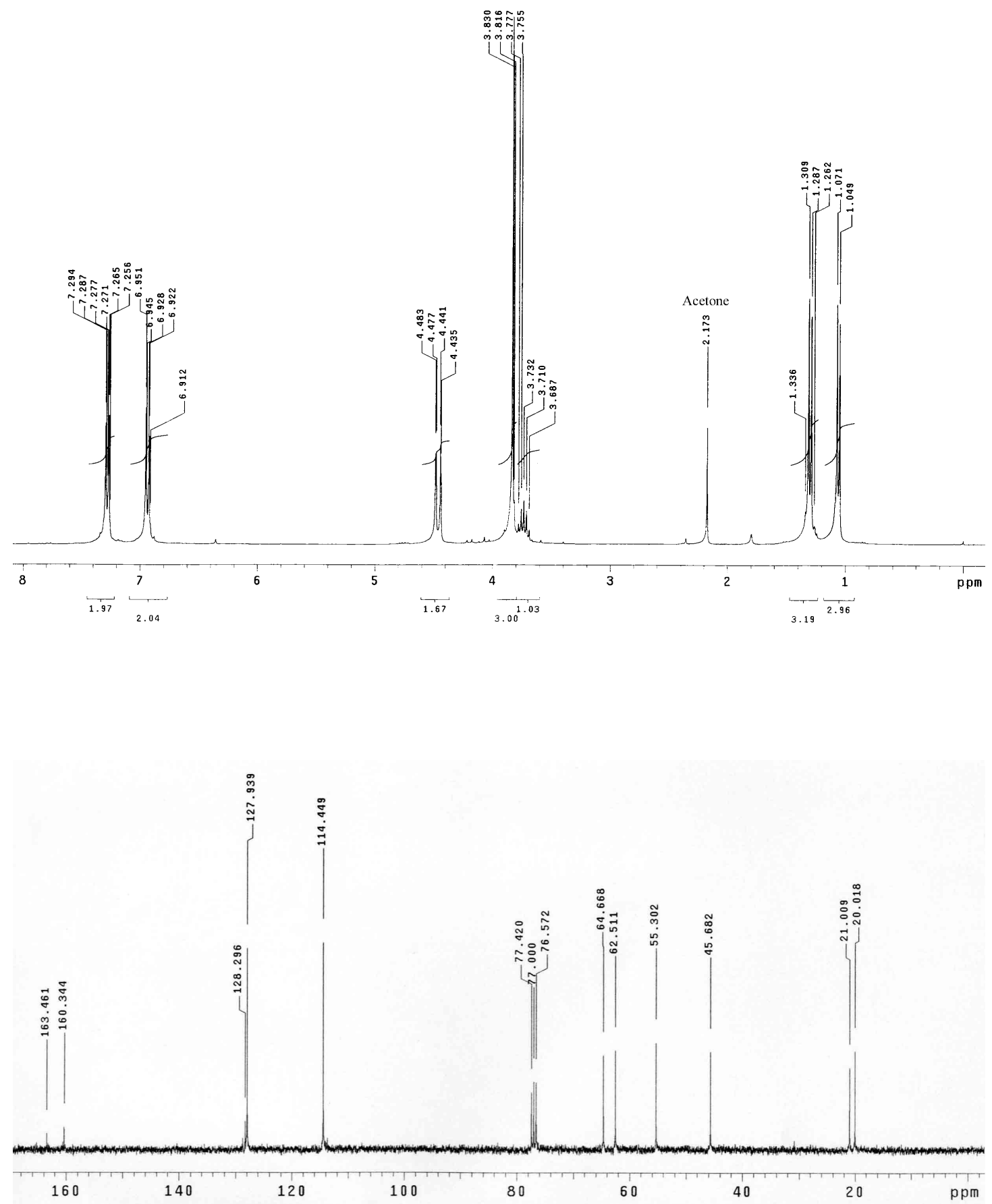
( \pm )-trans $-3-$ Chloro $^{-1-i s o p r o p y l-4-(4-n i t r o p h e n y l) a z e t i d i n-2-o n e ~(16 f) ~}$<smiles>O=C1[C@H](Cl)C(c2ccc([N+](=O)[O-])cc2)[C@H]1Cl</smiles>

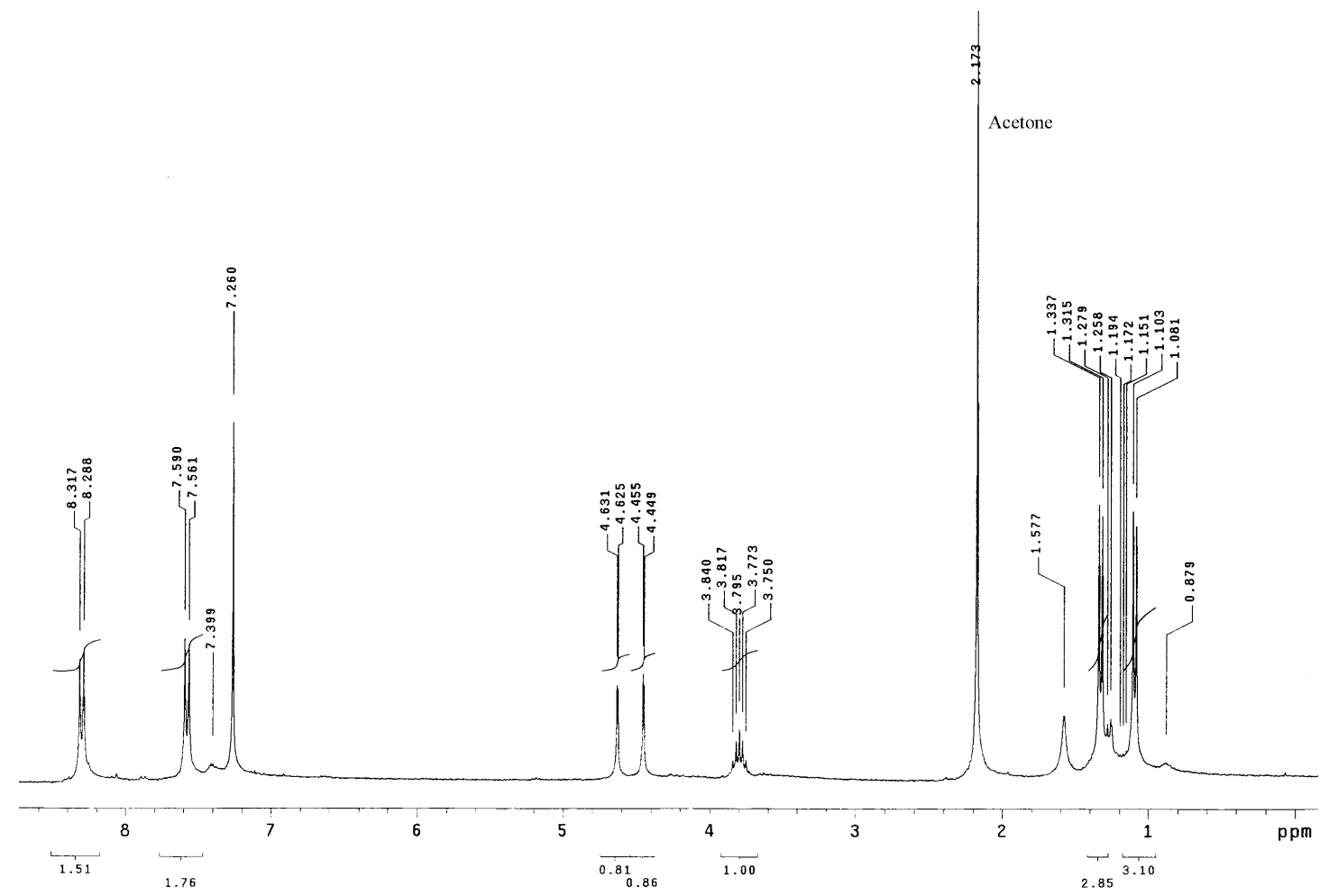

